ARTICLE

https://doi.org/10.1038/s41467-019-09417-1

\title{
MYB96 recruits the HDA15 protein to suppress negative regulators of ABA signaling in Arabidopsis
}

Hong Gil Lee ${ }^{1} \&$ Pil Joon Seo (10) ${ }^{1,2}$

Unlike activation of target genes in response to abscisic acid (ABA), how MYB96 transcription factor represses $A B A$-repressible genes to further enhance $A B A$ responses remains unknown. Here, we show MYB96 interacts with the histone modifier HDA15 to suppress negative regulators of early ABA signaling. The MYB96-HDA15 complex co-binds to the promoters of a subset of RHO GTPASE OF PLANTS (ROP) genes, ROP6, ROP10, and ROP11, and represses their expression by removing acetyl groups of histone $\mathrm{H} 3$ and $\mathrm{H} 4$ from the cognate regions, particularly in the presence of ABA. In support, HDA15-deficient mutants display reduced $A B A$ sensitivity and are susceptible to drought stress with derepression of the $R O P$ genes, as observed in the myb96-1 mutant. Biochemical and genetic analyses show that MYB96 and HDA15 are interdependent in the regulation of ROP suppression. Thus, MYB96 confers maximal $A B A$ sensitivity by regulating both positive and negative regulators of $A B A$ signaling through distinctive molecular mechanisms.

\footnotetext{
${ }^{1}$ Department of Chemistry, Seoul National University, Seoul 08826, Republic of Korea. ${ }^{2}$ Plant Genomics and Breeding Institute, Seoul National University, Seoul 08826, Republic of Korea. Correspondence and requests for materials should be addressed to P.J.S. (email: pjseo1@snu.ac.kr)
} 
S essile plants have evolved a remarkable ability to adapt to the environment, which is largely mediated by abscisic acid (ABA)-dependent signaling pathways ${ }^{1,2}$. ABA is recognized by plasma membrane-localized cell receptors, including PYRABACTIN RESISTANCE/PYRABACTIN RESISTANCE-LIKE/REGULATORY COMPONENT OF ABSCISIC ACID RECEPTOR (PYR/PYL/RCAR) family proteins ${ }^{3,4}$. These receptors constitute early ABA signaling modules, together with PROTEIN PHOSPHATASE 2Cs (PP2Cs) and subfamily 2 SNF1-RELATED PROTEIN KINASEs $(\mathrm{SnRK} 2 \mathrm{~s})^{5,6}$. In the presence of ABA, the PYR receptors inhibit PP2C activity and thereby derepress the catalytic functions of SnRK2 ${ }^{5,7}$. A number of transcription factors from bZIP, NAC, MYC, and MYB families are then activated to stimulate downstream ABA responses ${ }^{8,9}$.

The R2R3-type MYB96 transcription factor is a master transcriptional regulator that mediates a variety of plant responses to $\mathrm{ABA}$, including seed germination, drought tolerance, stomatal conductance, lateral root development, hormone biosynthesis, anthocyanin accumulation, and cuticular wax biosynthesis ${ }^{10-15}$. The ABA-inducible MYB96 transcription factor binds to the promoters of many ABA-responsive genes and usually activates expression to optimize plant growth and fitness under unfavorable conditions ${ }^{10-12}$. Notably, like many plant transcription factors $^{16}$, MYB96 has also been suspected to bind to the promoters of ABA-repressed genes, such as negative regulators of $\mathrm{ABA}$ signaling, and sometimes functions as a transcriptional repressor to further enhance plant responses to environmental stresses ${ }^{17-19}$. However, it remains unclear how MYB96 represses gene expression and what trans-factor mediates MYB96-dependent gene repression.

Two classes of signaling G proteins exist in eukaryotes: heterotrimeric $G$ proteins and monomeric Ras/Ras-like small GTPases $^{20,21}$. In particular, Rho family GTPases, which belong to the Ras superfamily, are likely major molecular switches that mediate diverse cellular responses to multiple extracellular signals in plants ${ }^{22}$. ROP (Rho of Plants)/RAC is the only subfamily of Rho family GTPases in plants ${ }^{20}$, and the Arabidopsis genome contains 11 ROP GTPases with four phylogenetic groups: group I (ROP8), group II (ROP9-ROP11), group III (ROP7), and group IV (ROP1-ROP6) 23,24 . A subset of ROP GTPases plays negative roles in ABA responses in Arabidopsis. For instance, overexpression of $R O P 2$ and $R O P 6$ reduces $A B A$ sensitivity during seed germination and stomatal closure ${ }^{25,26}$. The rop10 mutation enhances ABA-induced suppression of seed germination, primary root growth, and lateral root formation with increased expression of ABA-responsive genes ${ }^{27}$. ROP11 is also a negative regulator of multiple ABA responses, including seed germination, early seedling growth, stomatal closure, and drought responses ${ }^{28}$. Further, ROP11 acts at an early step of ABA signaling by interacting with $\mathrm{ABI} 1$, a receptor component, at the plasma membrane and protecting $\mathrm{ABI} 1$ phosphatase activity from inhibition by the ABA receptor complex ${ }^{29}$. The ROP signaling networks coordinate many downstream pathways to ensure large repertoires of output responses.

In this study, we find that MYB96 negatively regulates a subset of $R O P$ genes, which repress ABA signaling at the early stages of signal transduction. Repression of ROP genes by MYB96 requires the histone modifier HDA15. The MYB96 transcription factor specifically binds to promoters of the ROP6, ROP10, and ROP11 genes and recruits the HDA15 protein to catalyze $\mathrm{H} 3$ and $\mathrm{H} 4$ deacetylation at cognate sites. While MYB96 alone acts as a transcriptional activator, the MYB96-HDA15 complex can perform repressive functions in gene regulation. These results indicate that MYB96 acts as both a transcriptional activator and repressor to enhance ABA signaling.

\section{Results}

MYB96 interacts with HDA15. MYB96 is a pivotal regulator of ABA signaling and mediates a variety of physiological responses required for environmental stress adaptation in plants ${ }^{10-14,30,31}$. Consistent with MYB96 being a transcriptional activator, a number of ABA-inducible genes are primary targets of MYB96 ${ }^{11,12,14,30,31}$. However, accumulating evidence suggests that many negative regulators of $\mathrm{ABA}$ signaling may also be repressed in the myb96-ox overexpression mutant ${ }^{12}$, suggesting that MYB96 may further enhance ABA responses by directly repressing ABA-repressed genes.

We postulated that MYB96 may recruit additional trans-factor (s) to repress gene expression. To obtain clues to the identities of potential interacting factors, we performed yeast-two-hybrid $(\mathrm{Y} 2 \mathrm{H})$ screening. Preliminary analysis identified the HDA15 protein as an interaction partner of MYB96. To validate this observation, the MYB96 protein fused with the GAL4 activation domain $(\mathrm{AD})$ was coexpressed in yeast cells with the full-length HDA15 protein fused with the GAL4 DNA binding domain (BD). Growth on selective medium showed that MYB96 interacts with HDA15 (Fig. 1a). Deletion constructs further revealed that an Nterminal fragment of MYB96 containing the R2R3-MYB DNA $\mathrm{BD}$ was responsible for interaction with the N-terminal fragment of HDA15 (Fig. 1b, c). A zinc-finger motif in HDA15 might contribute to mediating the protein-protein interactions. Interactions of MYB96 and HDA15 were mutually specific. No other HDAC protein associated with MYB96 (Supplementary Figure 1). Likewise, other MYB transcription factors involved in ABA responses did not physically associate with HDA15 (Supplementary Figure 2).

To further support the in vivo interactions between MYB96 and HDA15, we conducted bimolecular fluorescence complementation (BiFC) analysis. The HDA15 sequence was translationally fused in-frame to the 5 -end of a gene sequence encoding the N-terminal half of yellow fluorescent protein (nYFP), and the MYB96 gene was fused in-frame to the 5'-end of a sequence encoding the C-terminal half of YFP (cYFP). The fusion constructs were then transiently coexpressed in Arabidopsis protoplasts. As a result, strong yellow fluorescence was detected in the nucleus for the MYB96-HDA15 combination, while no fluorescence signal was observed when empty vectors were coexpressed (Fig. 1d and Supplementary Figure 3).

We confirmed in planta interactions of MYB96 and HDA15 by coimmunoprecipitation (Co-IP). 35S:MYB96-MYC and 35S: HDA15-GFP constructs were coexpressed in leaf cells of Nicotiana benthamiana by Agrobacterium infiltration. Total protein extracts were immunoprecipitated with an anti-MYC antibody, which mainly pulls down the MYB96-MYC protein. Then immunoblot analysis was performed with an anti-green fluorescent protein (anti-GFP) antibody and the HDA15 fusion protein was detected (Fig. 1e), indicating in planta interactions between MYB96 and HDA15.

HDA15 promotes ABA signaling. Our results raised the possibility that HDA15 is involved in ABA signaling, possibly in connection with MYB96-regulated signaling networks. Thus we obtained T-DNA insertional mutants, hda15-1 and hda15-2, and produced HDA15-overexpressing HDA15-ox transgenic plants (Supplementary Figure 4) to examine ABA sensitivity in germination rate. In the absence of $A B A$, the germination rate of hda15-deficient mutant and HDA15-ox transgenic seeds was indistinguishable from wild-type seeds (Fig. 2a, b). However, in the presence of ABA, hda15 mutant seeds exhibited reduced sensitivity to ABA (Fig. 2a, b), similar to myb96-1 mutant seeds ${ }^{10,14}$. In contrast, higher ABA sensitivity was observed in 
a

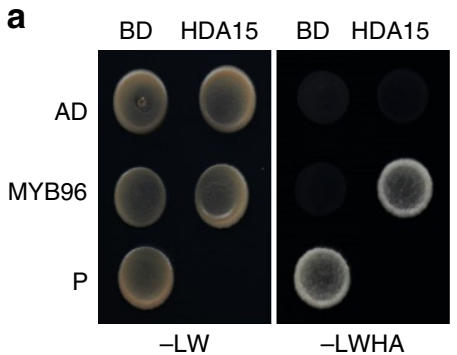

b

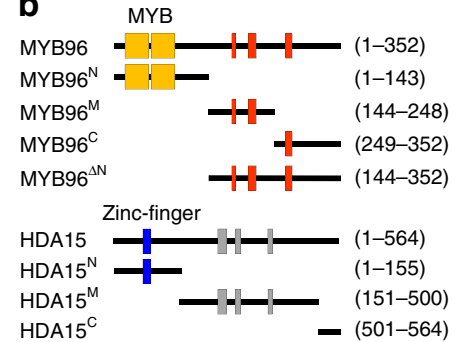

C
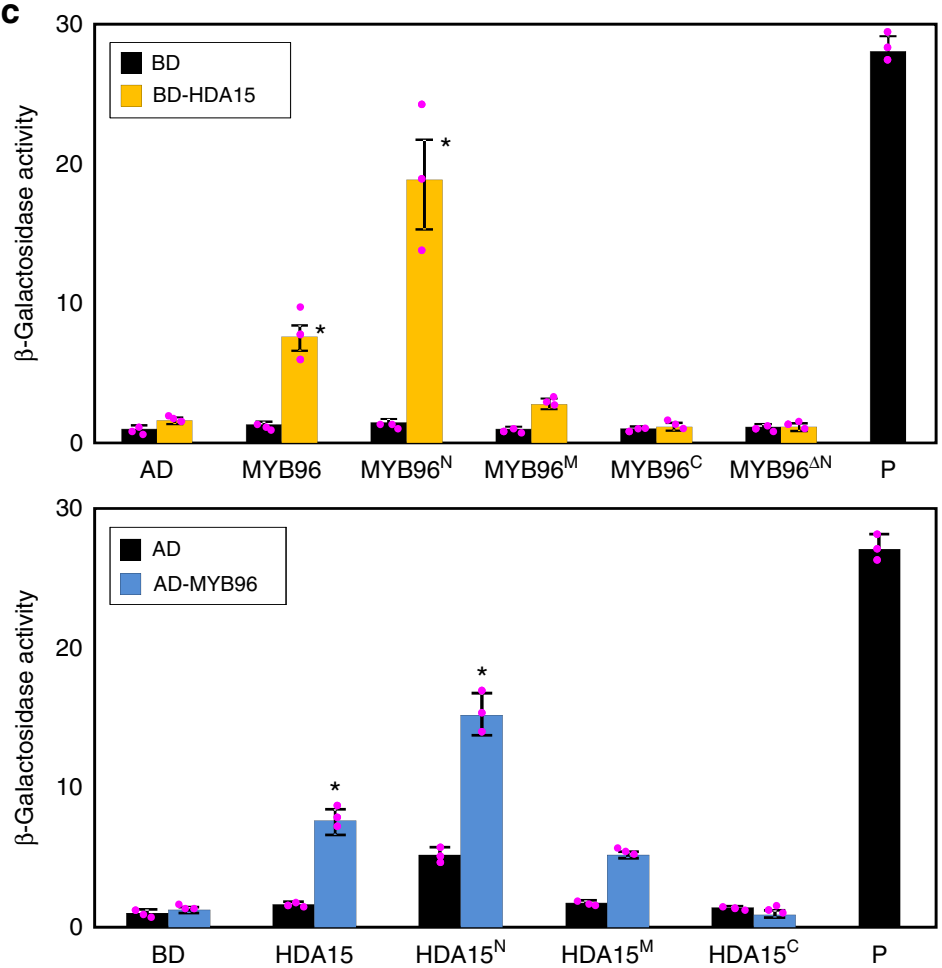

d

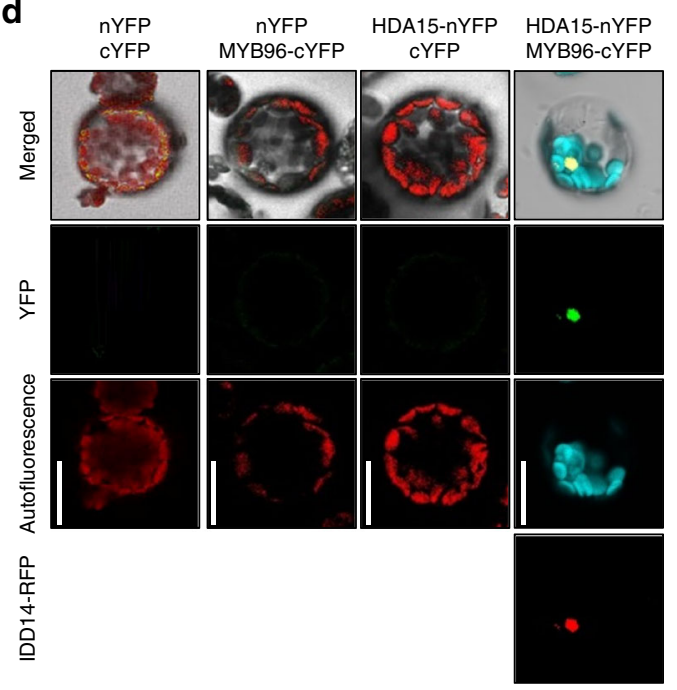

e
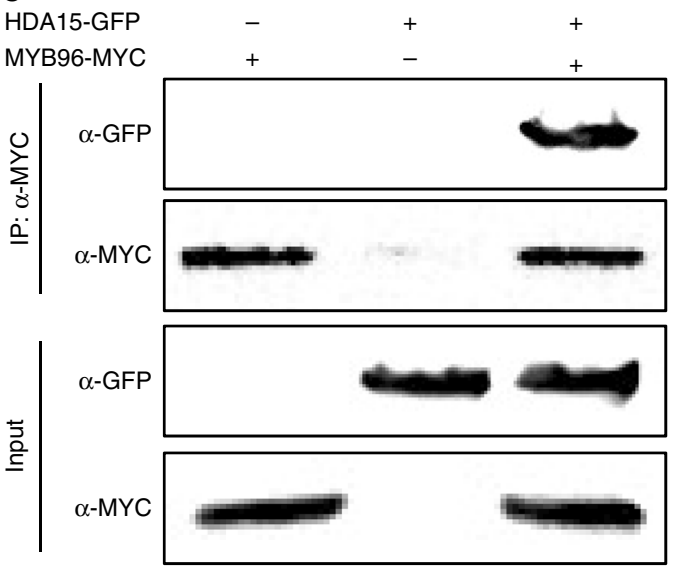

Fig. 1 Interaction of MYB96 with HDA15. a Yeast-two-hybrid $(Y 2 H)$ assays. $Y 2 H$ assays were performed with the HDA15 protein fused to the DNA binding domain (BD) of GAL4 and MYB96 fused to the transcriptional activation domain (AD) of GAL4. Interaction of the two proteins was examined by cell growth on selective media. -LWHA indicates Leu, Trp, His, and Ade drop-out plates. -LW indicates Leu and Trp drop-out plates. GAL4 was used as a positive control (P). b Deletion constructs of HDA15 and MYB96. Numbers indicate residue positions. Red and gray rectangles represent low complexity regions and histone deacetylase domains, respectively. $\mathbf{c}$ Interaction domain mapping. $\beta$-Galactosidase ( $\beta$-Gal) activity was quantified after growing yeast strains in liquid culture with 0 -nitrophenyl- $\beta$-D-galactopyranoside as a substrate. Three independent measurements of $\beta$-galactosidase activities were averaged and statistically analyzed by a Student's $t$ test $\left({ }^{\star} P<0.05\right)$. Bars indicate the standard error of the mean. $\mathbf{d}$ Bimolecular fluorescence complementation assays. Partial yellow fluorescent protein fusion constructs containing either HDA15 or MYB96 were transiently co-expressed in Arabidopsis protoplasts. The IDD14-RFP construct was used as a nuclear localization marker. Scale bars $=20 \mu \mathrm{m}$. e Coimmunoprecipitation (Co-IP) assays. Agrobacterium tumefaciens cells containing MYB96-MYC and HDA15-GFP constructs were injected into 3-week-old Nicotiana benthamiana leaves. Epitopetagged proteins were detected immunologically using corresponding antibodies. IP immunoprecipitation. Source data are provided as a Source Data file

HDA15-ox and myb96-ox transgenic seeds during germination (Fig. 2a, b). The differential sensitivity was more pronounced at higher concentrations of ABA (Supplementary Figure 5). Moreover, plant tolerance to drought stress, which is mainly conferred by $\mathrm{ABA}^{32}$, was also influenced by HDA15 (Fig. 2c). The hda15 mutant plants exhibited higher susceptibility to water deficit, whereas HDA15-ox transgenic plants were more tolerant to drought stress than wild-type plants (Fig. 2c, d), similar to MYB96-misexpressing plants (Supplementary Figure 6), suggesting that HDA15 positively regulates ABA responses.

Consistent with the positive role of HDA15 in ABA responses, the HDA15 gene was induced by ABA treatment (Fig. 2e and Supplementary Figure 7). Abiotic stress factors, including osmotic and cold stress, also promoted HDA15 expression (Supplementary Figure 7). In addition, the expression of some ABAresponsive genes was influenced by HDA15 activity. Even in the absence of exogenous ABA application, HDA15-ox transgenic plants exhibited increased transcript accumulation of ABAresponsive marker genes such as RESPONSIVE TO DESSICATION 22 (RD22), RD29A, and COLD-REGULATED 47 (COR47) $)^{33}$, whereas the hda15-1 mutant showed reduced transcript accumulation of these genes (Fig. 2f). Furthermore, ABA induction of the RD and COR genes was also impaired in the hda15-1 and myb96-1 mutants, regardless of plant ages (Fig. $2 \mathrm{~g}$ and Supplementary Figure 8). These results indicate that HDA15 is an ABA signaling component that promotes $\mathrm{ABA}$ responses.

MYB96 and HDA15 act synergistically in ABA signaling. Considering that MYB96 interacts with HDA15 and that they have overlapping functions in ABA signaling, MYB96 and HDA15 may act together in the control of ABA sensitivity. We 
a

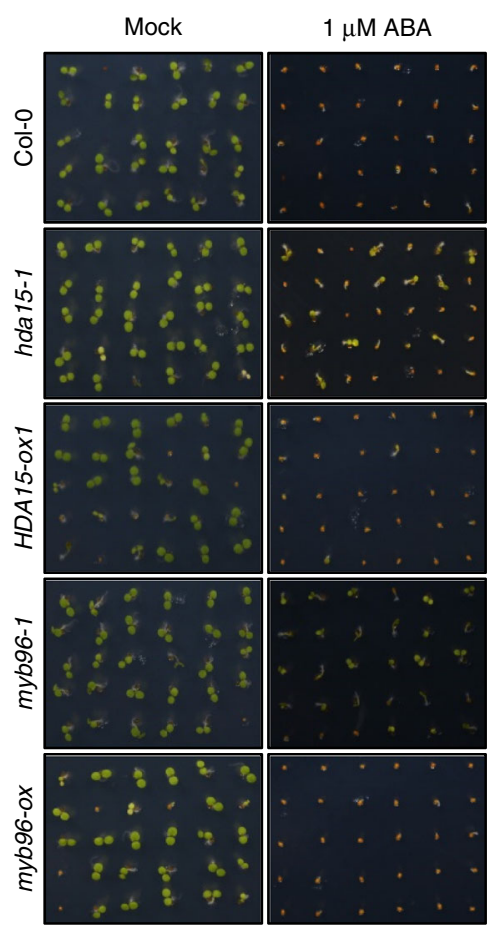

C

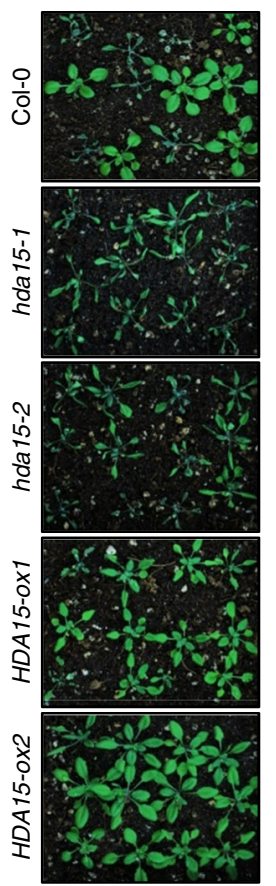

d

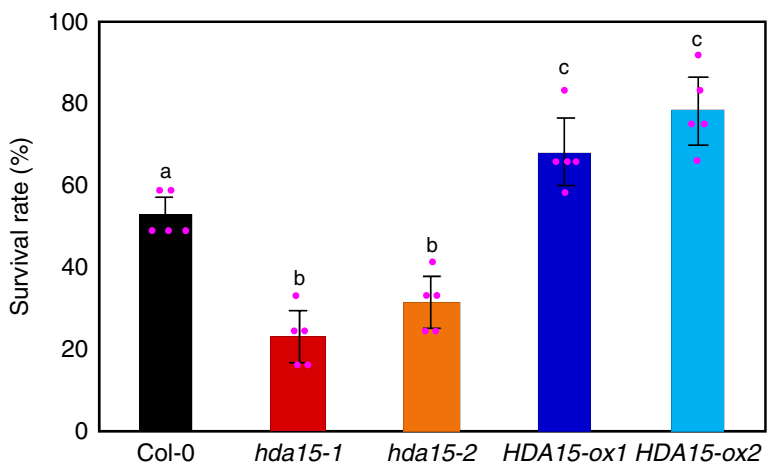

e

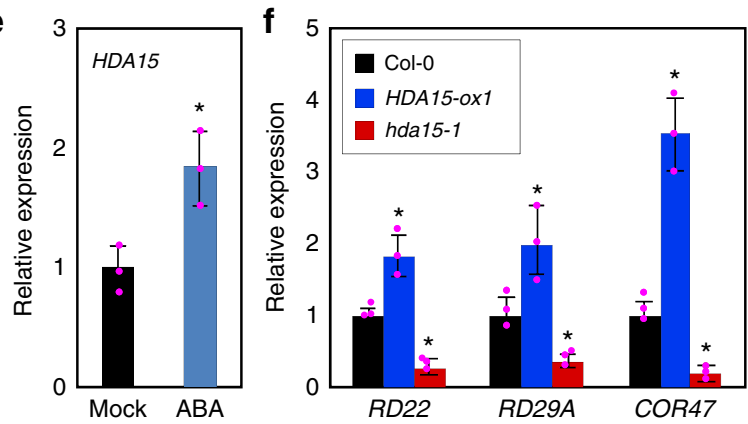

g
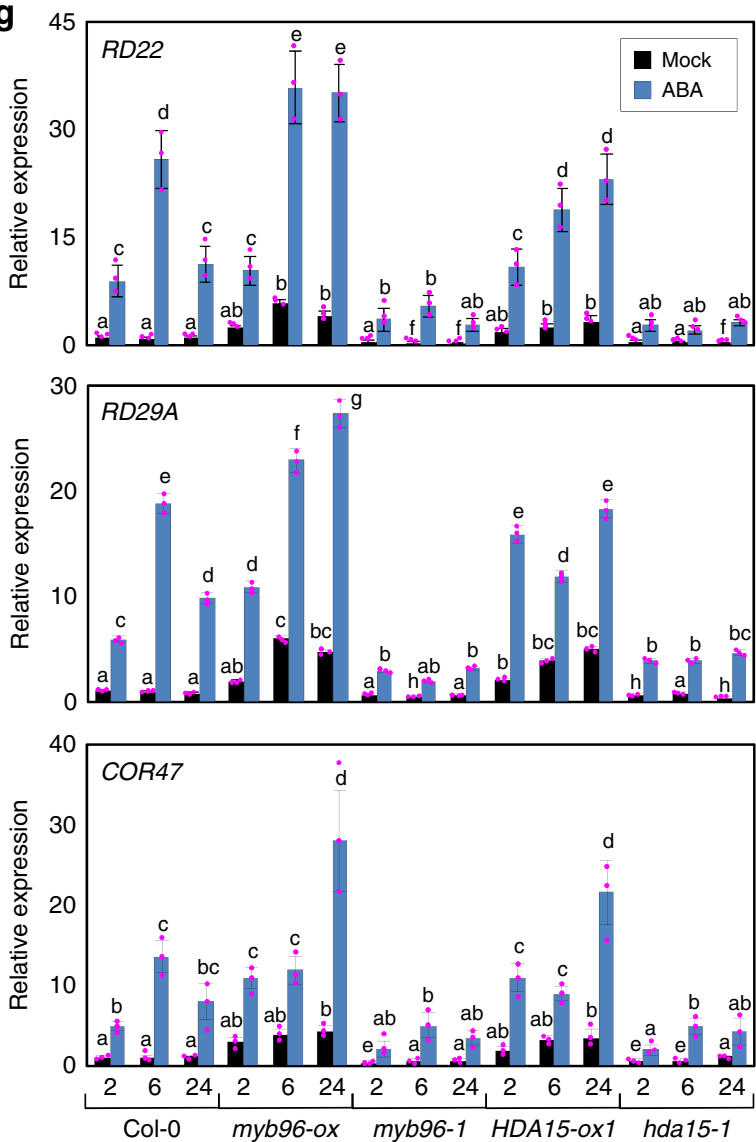

(Fig. 3a), suggesting that they work together especially at high concentrations of ABA.

We then analyzed the genetic relationship between MYB96 and HDA15. To this end, the myb96-1hda15-1 double mutant was generated to analyze $\mathrm{ABA}$ responses including seed germination and drought tolerance. In the presence of $\mathrm{ABA}$, seed germination 
Fig. 2 Abscisic acid (ABA) sensitivity of HDA15-ox transgenic and hda15-deficient plants. a Altered ABA sensitivity of HDA15-ox and hda15-1 seeds. Seeds were germinated and grown on MS-medium supplemented with $1 \mu \mathrm{M} \mathrm{ABA}$ under long-day (LD) conditions. Photographs were taken 4 days after cold stratification (DAC). myb96-1 and myb96-ox mutant seeds were used as controls. b Germination percentage. The percentage of seed germination of the indicated genotypes grown on different concentrations of ABA was quantified after the end of stratification ( $h$ ). Radicle emergence was used as a morphological marker for germination. At least 30-50 seeds per genotype were measured in each replicate. Biological triplicates were averaged. Bars indicate the standard deviation of the mean. c Drought tolerance of HDA15-ox and hda15 mutant plants. d Survival rate. Three independent biological replicates were averaged, and statistical significance of the measurements was analyzed by analysis of variance (ANOVA) (one-way ANOVA with Fisher's post hoc test, ${ }^{\star} P<0.05$ ). Different letters represent a significant difference at $P<0.05$. Bars indicate the standard deviation of the mean. e Effects of $A B A$ on HDA15 expression. Two-week-old plants grown under LDs were transferred to half-strength MS-liquid medium supplemented with $20 \mu \mathrm{M} A B A$ and incubated for $6 \mathrm{~h}$. Transcript accumulation was analyzed by quantitative real-time reverse transcriptase-PCR. Three independent biological replicates were averaged, and statistical significance of the measurements was analyzed by a Student's $t$ test $\left({ }^{\star} P<0.05\right)$. Bars indicate the standard error of the mean. f Expression of ABA-responsive genes in HDA15-ox and hda15-1. Three independent biological replicates were averaged, and statistically significant differences between the wild-type and transgenic or mutant plants are indicated by asterisks (Student's $t$ test, ${ }^{\star} P<0.05$ ). $\mathbf{g}$ HDA15 regulation of ABA induction of $A B A$ marker genes. Two-week-old seedlings grown under LDs were transferred to MS-liquid medium supplemented with $20 \mu \mathrm{M} A B A$ and incubated for the indicated time period $(h)$. Three independent biological replicates were averaged, and statistical significance of the measurements was analyzed by ANOVA (one-way ANOVA with Fisher's post hoc test, ${ }^{\star} P<0.05$ ). Different letters represent a significant difference at $P<0.05$. Source data are provided as a Source Data file

rate was significantly delayed in wild type, whereas myb96-1 and hda15-1 mutant seeds exhibited reduced sensitivity to ABA (Fig. 3b). Notably, the myb96-1hda15-1 double mutant showed further reduction in ABA sensitivity compared to myb96-1 and hda15-1 seeds (Fig. 3b), particularly at a high concentration of ABA (Fig. 3b). Similarly, the myb96-1hda15-1 double mutant also exhibited a higher susceptibility to drought stress than myb96-1 and hda15-1 single mutants (Fig. 3c). The reduced sensitivity to ABA in myb96-1hda15-1 was supported by expression of the RD and $C O R$ genes. $\mathrm{ABA}$ induction of $R D$ and $C O R$ genes was compromised in myb96-1 and hda15-1 mutants and more profoundly in the myb96-1hda15-1 double mutant (Fig. 3d and Supplementary Figure 8). These results indicate that MYB96 and HDA15 act synergistically to confer ABA sensitivity at least in the processes of seed germination and drought tolerance.

MYB96 recruits HDA15 to the ROP promoters. Previous microarray data assumed that MYB96 represses some negative regulators of ABA signaling ${ }^{12}$. To test this possibility, we examined transcript accumulation of negative regulators of ABA signaling in wild-type, myb96-ox, and myb96-1 seedlings, including ABI1, HIGHLY ABA-INDUCED PP2C GENE 2 (HAI2), HYPERSENSITIVE TO ABA 1 (HAB1), ABI3-INTERACTING PROTEIN 2 (AIP2), PLANT U-BOX 9 (PUB9), MORE AXILLARY GROWTH 2 (MAX2), DWD HYPERSENSITIVE TO ABA 1 (DWA1), DWA2, and ROP genes. These genes are known as negative regulators of $\mathrm{ABA}$ signaling ${ }^{34-37}$, and their genetic mutants display enhanced ABA responses, such as delayed germination, reduced lateral root length, and increased drought tolerance ${ }^{38-40}$. Quantitative real-time reverse transcriptase-PCR (RT-qPCR) analysis showed that most of the genes were uninfluenced by MYB96, except for a subset of ROP genes, ROP6, ROP10, and ROP11 (Fig. 4a; Supplementary Figure 9), which are known to negatively regulate early ABA signaling $26,29,41$. These $R O P$ genes were specifically and significantly activated in myb96-1 but reduced in myb96-ox (Fig. 4a).

Based on the mutual interaction of MYB96 and HDA15 and the transcriptional repressor activity of HDA1 $5^{42,43}$, we hypothesized that MYB96 works together with HDA15 to repress the $R O P$ genes. Although HDA15 broadly influenced the expression of $R O P$ genes, the three $R O P$ genes were specifically upregulated in the hda15-1 mutant (Fig. 4b). To further estimate the relevance of MYB96 and HDA15 in ABA-dependent ROP suppression, we tested transcript levels of ROP6, ROP10, and ROP11 in myb96-1, hda15-1, and myb96-1hda15-1 mutant seedlings treated with $\mathrm{ABA}$. The ROP genes were repressed in response to ABA in wild type, but ABA repression of $R O P$ genes was impaired, and higher expression of ROP genes was observed in the myb96-1, hda15-1, and myb96-1hda15-1 mutants (Fig. 4c). Although MYB96 and HDA15 were synergistic in the control of ABA responses (Fig. 3a-d), they both were required at least for the suppression of $R O P$ expression in response to ABA (Fig. 4c).

We then asked whether the ROP6, ROP10, and ROP11 genes are direct regulatory targets of MYB96 and HDA15. To answer this question, we employed 35S:MYB96-MYC and 35S:HDA15GFP transgenic plants ${ }^{43}$ and performed chromatin immunoprecipitation (ChIP) using the corresponding antibodies. ChIPqPCR analysis showed that MYB96 binds to specific regions of the $R O P$ promoters (Fig. 5a), and consistently, HDA15 bound to a similar region at each gene promoter (Fig. 5b). The binding regions of MYB96 and HDA15 at the ROP chromatins were maintained in transgenic plants expressing native promoterdriven constructs (Supplementary Figure 10).

These observations suggest that MYB96 most likely binds to specific promoter regions and recruits HDA15 that has no DNAbinding specificity. In support, while the hda15-1 mutation did not influence MYB96 binding to the ROP loci (Fig. 5a), binding of HDA15 to the ROP promoters was markedly reduced in the myb96-1 background (Fig. 5b). These results indicate that HDA15 requires MYB96 to associate with the $R O P$ promoters and repress expression.

MYB96-HDA15 complex catalyzes histone deacetylation at ROP genes. Since HDA15 represses gene expression by promoting $\mathrm{H} 3$ and $\mathrm{H} 4$ deacetylation ${ }^{42,43}$, we asked whether the MYB96-HDA15 complex catalyzes removal of $\mathrm{H} 3$ and $\mathrm{H} 4$ acetylation ( $\mathrm{H} 3 \mathrm{ac}$ and $\mathrm{H} 4 \mathrm{ac}$ ) at the $R O P$ promoters. To this end, ChIP assays using anti-H3ac and anti-H4ac antibodies were performed. In the presence of $\mathrm{ABA}$, acetylation levels of $\mathrm{H} 3$ and $\mathrm{H} 4$ at the binding regions on the $R O P$ promoters were significantly reduced in the wild-type background (Fig. 6a, b). However, ABA repression of $\mathrm{H} 3 \mathrm{ac}$ and $\mathrm{H} 4 \mathrm{ac}$ accumulation at the $R O P$ promoters was compromised in myb96-1, hda15-1, and myb96-1hda15-1 mutants (Fig. 6a, b), consistent with the ROP transcript levels (Fig. 4c). The histone modifications were most likely propagated into distal regions of the promoters, and farther promoter regions were also modified (Supplementary Figure 11), similar to core-binding regions (Fig. 6a, b). As a negative control, we also analyzed accumulation of $\mathrm{H} 3 \mathrm{ac}$ and $\mathrm{H} 4 \mathrm{ac}$ at the gene body regions of the ROP genes (Fig. 5). As expected, histone acetylation levels were unchanged in myb96-1, hda15-1, and myb96-1hda15-1 mutants, regardless of ABA treatment (Fig. 6a, 
a

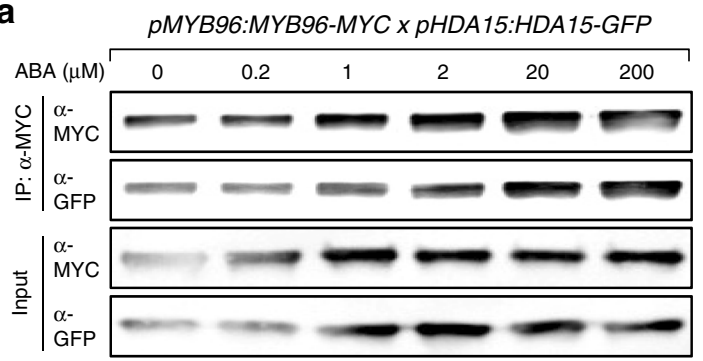

b
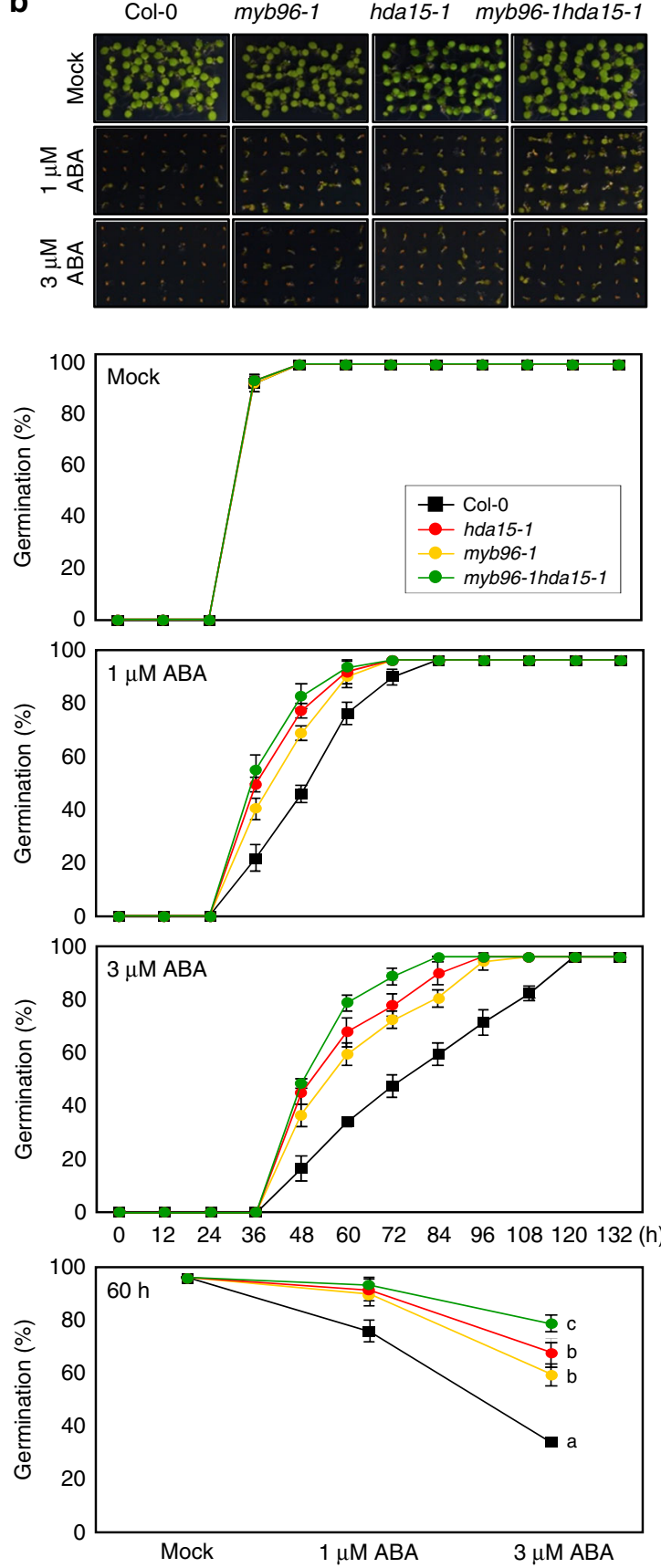

b). These results indicate that MYB96 binds to specific regions of the ROP promoters and recruits HDA15 to remove the acetyl groups of $\mathrm{H} 3$ and $\mathrm{H} 4$ at the promoter regions.

Considering the requirement of MYB96 for HDA15 recruitment (Fig. 5), we wanted to know whether MYB96 is also
C
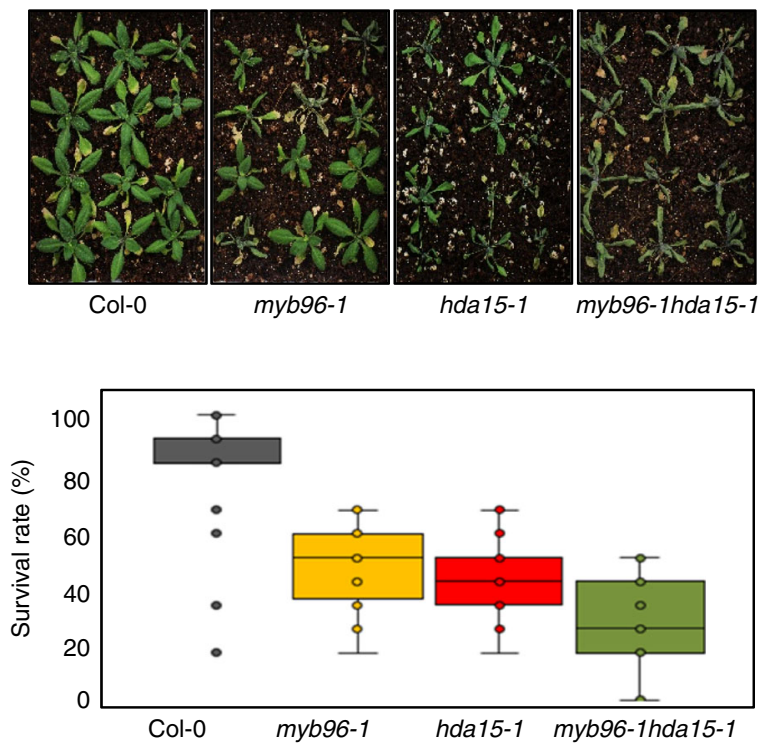

d
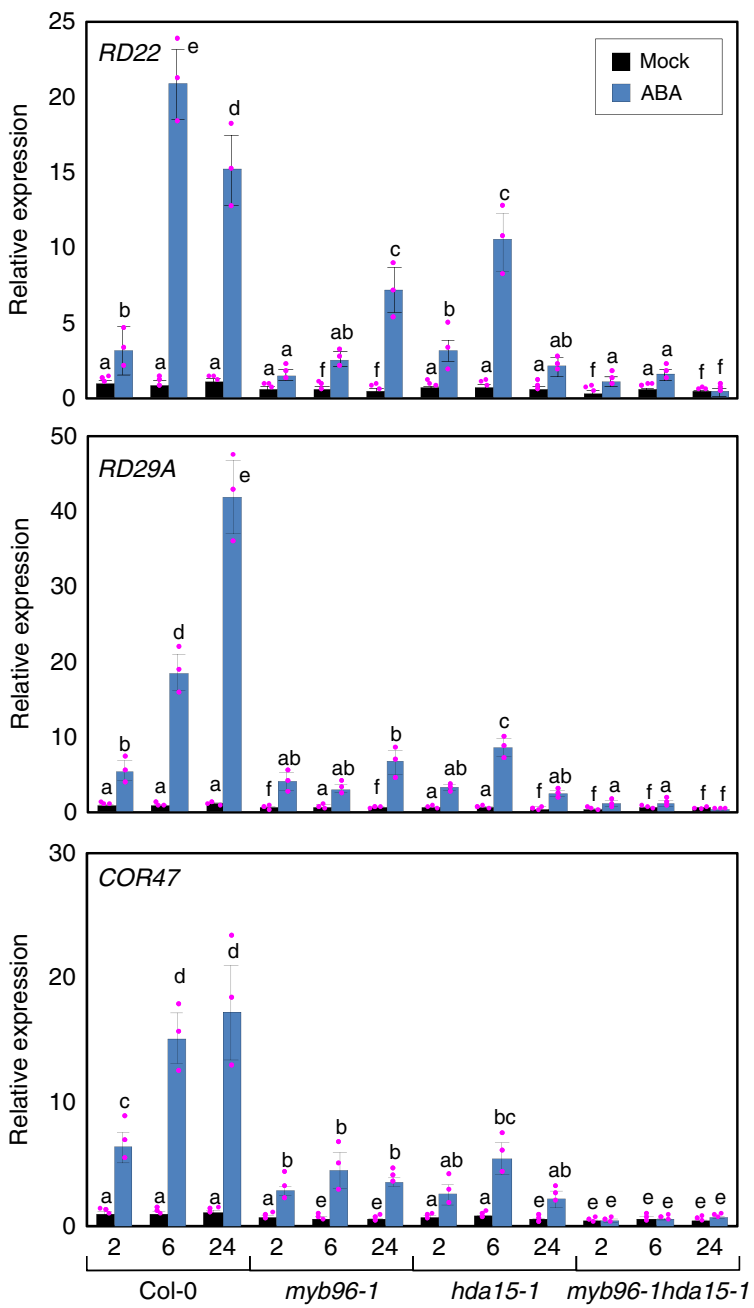

required for histone deacetylation at the $R O P$ promoters. We performed ChIP assays with anti-H3ac antibody using 35S: HDA15-GFP/myb96-1 plants. While H3ac levels at the ROP promoters were significantly reduced in 35S:HDA15-GFP transgenic plants compared with wild-type plants even in the absence 
Fig. 3 Synergistic interactions between MYB96 and HDA15. a Effects of abscisic acid (ABA) on interactions between MYB96 and HDA15. Two-week-old seedlings were used for treatment with different concentrations of $A B A$. Epitope-tagged proteins were precipitated and detected immunologically using the corresponding antibodies. IP immunoprecipitation. b Effects of ABA on seed germination in myb96-1hda15-1. The percentage of seed germination of the indicated genotypes grown on different concentrations of ABA was quantified after the end of stratification ( $h$ ). Biological triplicates were averaged. Bars indicate the standard deviation of the mean. c Drought tolerance of myb96-1hda15-1 plants. Two-week-old plants were treated for drought stress by stopping watering for additional 2 weeks. Three independent biological replicates were averaged. d ABA induction of ABA marker genes in myb96-1hda15-1. Two-week-old seedlings grown under long days were transferred to MS-liquid medium supplemented with $20 \mu \mathrm{M}$ ABA and incubated for the indicated time period (h). Three independent biological replicates were averaged, and statistical significance of the measurements was analyzed by analysis of variance (ANOVA) (one-way ANOVA with Fisher's post hoc test, ${ }^{\star} P<0.05$ ). Different letters represent a significant difference at $P<0.05$. Source data are provided as a Source Data file

of ABA, suppression was diminished in 35S:HDA15-GFP/myb961 (Fig. 6c). Consistently, in the absence of $\mathrm{ABA}$, the ROP genes were repressed in 35S:HDA15-GFP transgenic plants, but this transcriptional repression was restored in 35S:HDA15-GFP/ myb96-1 plants (Fig. 6d). These results indicate that the HDA15 protein binds to the $R O P$ promoters and catalyzes histone deacetylation in a MYB96-dependent manner.

MYB96 represses ROP genes in an HDA15-dependent manner. We next asked whether MYB96 repression of ROP genes also depends on HDA15. To answer this question, we carried out transient expression assays using Arabidopsis protoplasts. Reporter constructs, in which the ROP and KETOACYL-COA SYNTHASE (KCS) promoter sequences were fused with the minimal $35 \mathrm{~S}$ promoter, were coexpressed with an effector construct overexpressing MYB96 in Arabidopsis mesophyll protoplasts isolated from wild-type and $h d a 15-1$ leaves (Fig. 7a). Reporter GUS activity measurement revealed that MYB96 significantly repressed $R O P$ promoter activity in the wild-type background, but the repressive function was impaired in the hda15-1 background (Fig. 7b). The impaired MYB96 function in hda15-1 was recovered by complementation of HDA15 (Supplementary Figure 12).

In addition, as a control, we also examined whether HDA15 affects MYB96-dependent ABA-inducible genes. Expression of MYB96 alone was sufficient to activate the KCS genes, which are the primary target genes directly activated by $\mathrm{MYB}^{12}{ }^{12}$, and the hda15-1 mutation did not influence MYB96 regulation of ABAinducible genes (Fig. 7b). Furthermore, coexpression of MYB96 and HDA15 in wild-type protoplasts also did not influence KCS1 expression (Supplementary Figure 12), suggesting that HDA15selectively inhibits MYB96-repressed genes.

We also measured $\mathrm{H} 3 \mathrm{ac}$ accumulation at the $\mathrm{ROP}$ promoters in 35S:MYB96-MYC and 35S:MYB96-MYC/hda15-1 plants. As expected, $\mathrm{H} 3 \mathrm{ac}$ levels at the $R O P$ promoters were reduced in 35S:MYB96-MYC even in the absence of ABA, but reduction in H3ac levels was impaired in the hda15-1 background (Fig. 7c). Transcript accumulation of the ROP genes further supported these observations. Reduction of the ROP genes in 35S:MYB96MYC was restored in 35S:MYB96-MYC/hda15-1 (Fig. 7d). These observations indicate that the role of MYB96 in ROP repression depends on HDA15. Overall, the MYB96-HDA15 complex represses the $R O P$ genes, which are negative regulators of $\mathrm{ABA}$ signaling, through histone deacetylation to strongly promote ABA responses (Fig. 8).

\section{Discussion}

Transcriptional responses to environmental challenges are usually linked to chromatin regulation ${ }^{44,45}$. Accumulating evidence has shown that genome-wide chromatin modification/remodeling accompanies water-deficit stress ${ }^{46-49}$. For instance, global changes in histone variant H2A.Z deposition occur especially in stressresponsive genes, and the $\operatorname{arp} 6$ mutant exhibits higher sensitivity to osmotic stress with hyperactivation of stress-responsive genes ${ }^{50}$.
Consistently, a significant number of chromatin modifiers/ remodelers are differentially expressed in response to $\mathrm{ABA}$ and/ or drought stress to confer appropriate responses to environmental disadvantages ${ }^{50-54}$. As a supporting evidence, the Arabidopsis SWI/SNF chromatin-remodeling ATPase BRAHMA (BRM) represses ABA responses by directly promoting occupancy of the +1 nucleosome at the transcription start site of the $A B I 5$ locus in the absence of $\mathrm{ABA}^{55}$. ABI5 expression is derepressed in $\mathrm{brm}$ mutants with reduction of the +1 nucleosome occupancy ${ }^{55}$, and consistently, brm mutants exhibit ABA hypersensitivity 55 . Moreover, BRM interacts with SnRK2s and clade A PP2Cs, and BRM function is further shaped by its phosphorylation state ${ }^{56}$. When ABA levels increase, SnRK2s are activated and clade A PP2Cs are inhibited by PYR/PYL ABA receptors. ABA-activated SnRK2s phosphorylate BRM, which leads to inhibition of BRM activity and thereby $A B I 5$ induction. Under normal growth conditions with low ABA levels, dephosphorylation of BRM by PP2Cs restores BRM activity and represses $A B I 5$ expression $^{56}$.

Several histone modifiers are also involved in abiotic stress responses. Two evolutionarily conserved Arabidopsis Trithorax Group proteins (TrxGs), ARABIDOPSIS TRITHORAX 4 (ATX4) and ATX5, directly bind to the ABA-HYPERSENSITIVE GERMINATION 3 (AHG3) locus, which acts as a negative regulator in $\mathrm{ABA}$ signaling, and regulate $\mathrm{H} 3 \mathrm{~K} 4 \mathrm{me} 3$ deposition to activate expression ${ }^{57}$. Accordingly, the atx 4 and at $x 5$ single and the at $x 4$ at $x 5$ double mutants exhibit higher drought tolerance and $\mathrm{ABA}$ hypersensitive phenotypes ${ }^{57}$. The RPD3-type histone deacetylases HDA6 and HDA19 also regulate the expression of ABA and abiotic stress-responsive genes by forming huge transcriptional repressor complexes, together with HISTONE DEACETYLATION COMPLEX 1 (HDC1), MULTICOPY SUPRESSOR OF IRA 1 (MSI1), ETHYLENE RESPONSE FACTOR 7 (ERF7), HISTONE DEACETYLASE 2C (HD2C), and SWIINDEPENDENT 3 (SIN3) in Arabidopsis ${ }^{19,58-61}$. These complexes usually repress expression of ABA-related genes by promoting histone deacetylation at cognate loci. Mutations of the components result in hyperactivation of ABA-responsive genes and thus hypersensitivity to $\mathrm{ABA}$ and salt stress and increased tolerance of osmotic stress $19,58-60,62,63$.

In this study, we found that HDA15 acts as a positive regulator of $\mathrm{ABA}$ signaling by repressing $R O P$ genes, which are pivotal repressors of early ABA signaling $26,27,29,41$. Binding selectivity of HDA15 to the ROP loci is defined by the MYB96 transcription factor. In the presence of $A B A$, the MYB96-HDA15 complex co-binds to the ROP promoters and removes acetyl groups of histone $\mathrm{H} 3$ and $\mathrm{H} 4$ to stimulate $\mathrm{ABA}$ responses. Consistently, hda15 and myb96 mutants exhibit reduced ABA sensitivity, whereas transgenic plants overexpressing HDA15 and MYB96 are hypersensitive to ABA. Overall, ABA signaling is shaped by multiple layers of regulation $^{64}$, and epigenetic mechanisms are a key regulatory scheme in plant stress responses. 

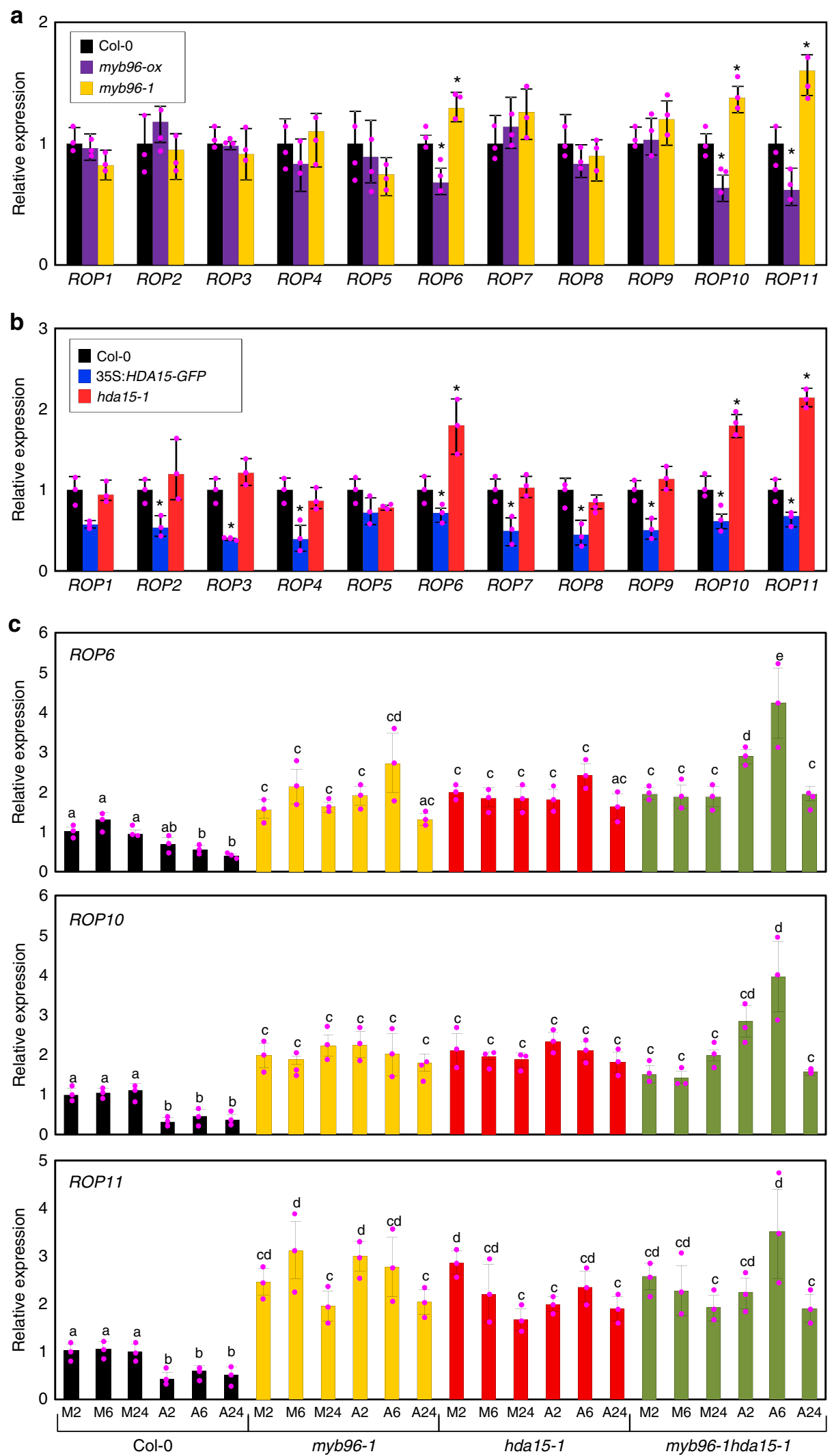

MYB96 regulates early signaling components of ABA pathways, including the ROP genes. Consistently, MYB96 has a strong impact on plant tolerance to abiotic stresses ${ }^{10,12-14,31}$ and regulates a variety of $\mathrm{ABA}$ responses, including lateral root development, stomatal movement, hormone biosynthesis, and cuticular wax biosynthesis ${ }^{10,12-14,31}$. Like many plant transcription factors,
MYB96 functions as a transcriptional repressor for some promoters but an activator for others. Our preliminary genome-wide identification of direct binding sites of MYB96 provides support that MYB96 binds to promoters of both ABA-repressed and ABAinduced genes. MYB96 transcriptional activity would be determined in part by trans-factors, such as histone-modifying enzymes. In this 
Fig. 4 MYB96 and HDA15 regulate expression of Rho of Plants (ROP) genes. a Transcript accumulation of ROP genes in wild type, myb96-ox, and myb96-1. b Transcript accumulation of ROP genes in wild type, 35S:HDA15-GFP, and hda15-1. In a, b, 2-week-old seedlings grown under long days (LDs) were harvested for total RNA isolation. Statistically significant differences between the wild-type and transgenic or mutant plants (Student's $t$ test, ${ }^{\star} P<0.05$ ) are indicated by asterisks. c Effects of abscisic acid (ABA) on the expression of ROP genes in myb96-1, hda15-1, and myb96-1hda15-1. Two-week-old seedlings grown under LDs were transferred to MS-liquid medium supplemented with $20 \mu \mathrm{M} \mathrm{ABA}$ and incubated for the indicated time period ( $h$ ). Transcript accumulation was analyzed by quantitative real-time reverse transcriptase-PCR. Three independent biological replicates were averaged, and statistical significance of the measurements was analyzed by analysis of variance (ANOVA) (one-way ANOVA with Fisher's post hoc test, ${ }^{\star} P<0.05$ ). $M$ mock, $A$ ABA. Different letters represent a significant difference at $P<0.05$. Source data are provided as a Source Data file

a
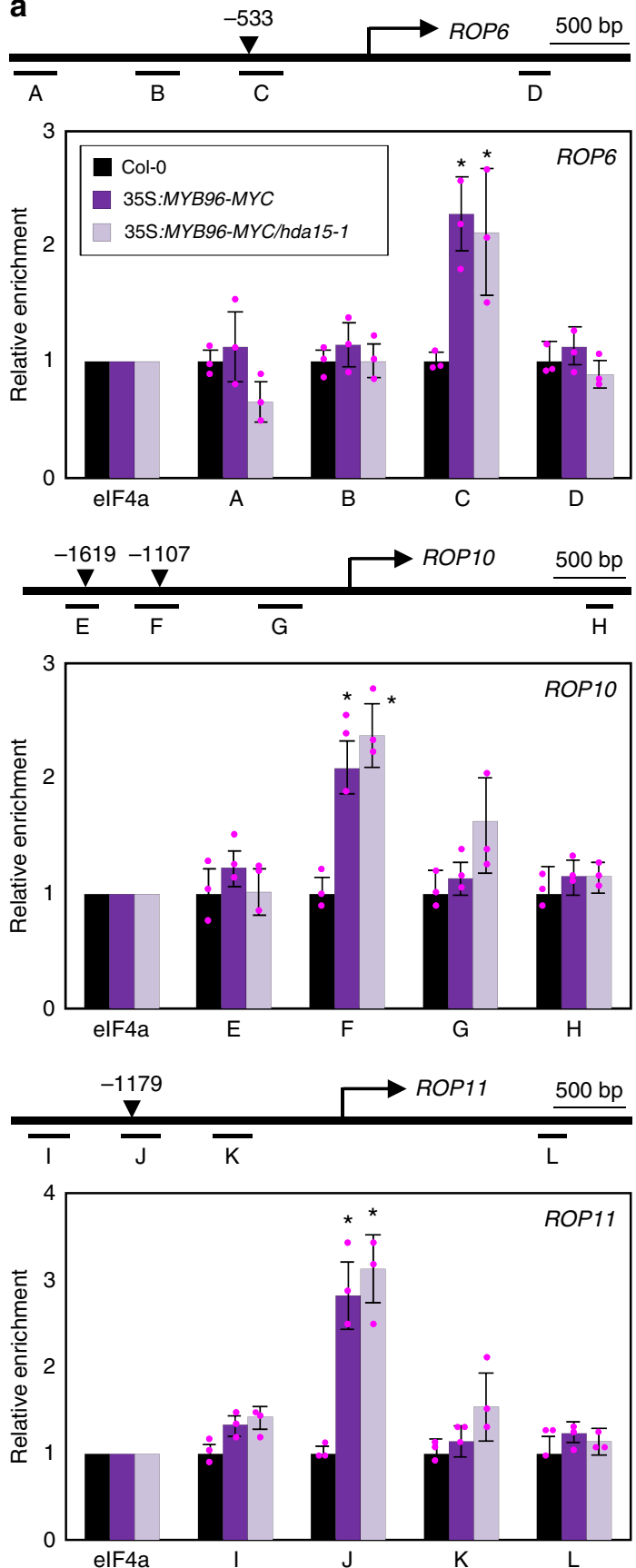

b
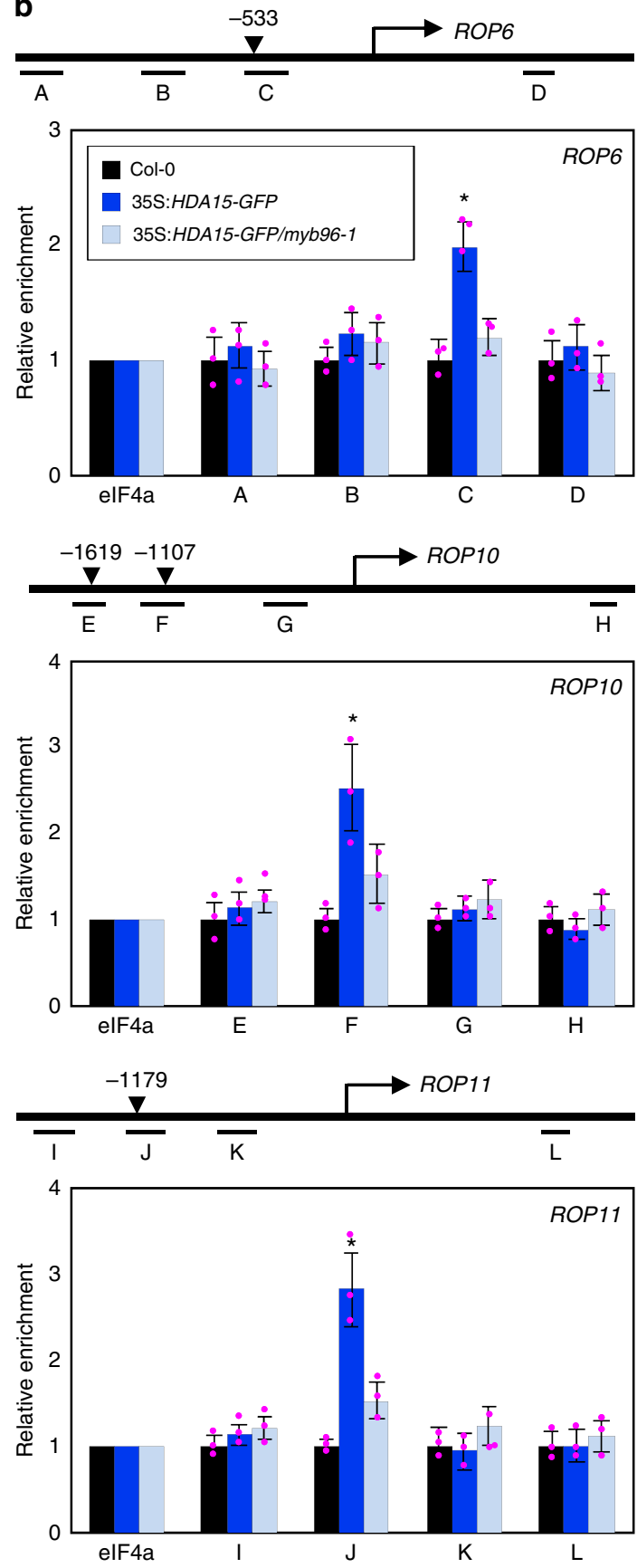

Fig. 5 MYB96 and HDA15 co-bind to the ROP promoters. In $\mathbf{a}, \mathbf{b}$, putative MYB-binding sites are indicated by arrowheads. Black underbars indicate the regions of PCR amplification after chromatin immunoprecipitation (ChIP). ChIP-quantitative PCR assays were conducted to examine enrichment of MYB96 and HDA15 at ROP loci. Three independent biological replicates were averaged, and statistical significance of the measurements was analyzed by Student's $t$ test $\left({ }^{\star} P<0.05\right)$. Bars indicate the standard error of the mean. a Binding of MYB96 to ROP loci. $\mathbf{b}$ Binding of HDA15 to ROP loci in a MYB96-dependent manner. Source data are provided as a Source Data file 

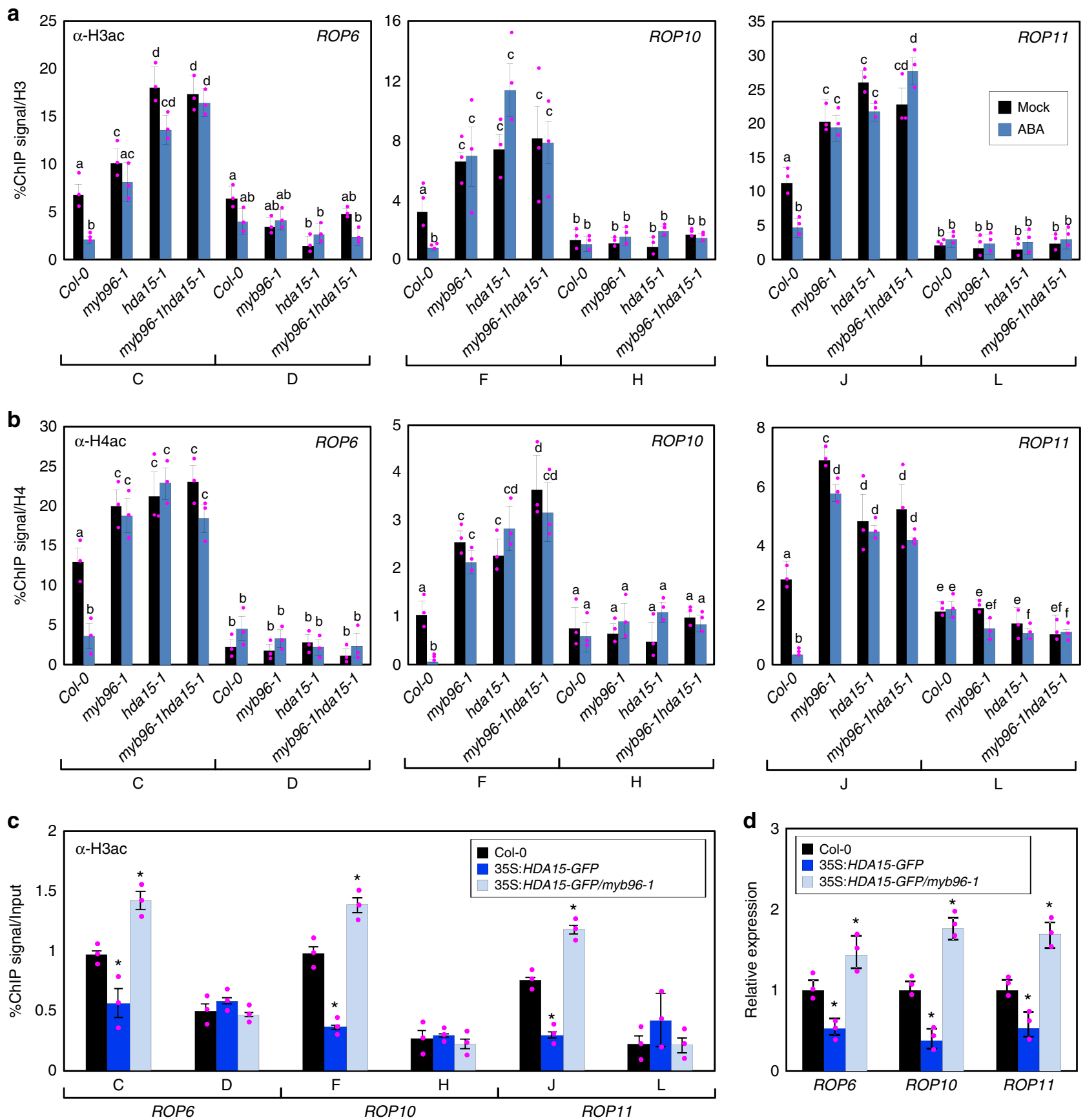

Fig. 6 HDA15 and MYB96 co-regulate histone deacetylation at ROP loci. a Effects of abscisic acid (ABA) on H3ac accumulation at the ROP promoters in myb96-1, hda15-1, and myb96-1hda15-1 mutants. b Effects of ABA on H4ac accumulation at the ROP promoters. In a, b, 2-week-old seedlings grown under long days (LDs) were transferred to MS-liquid medium supplemented with $20 \mu \mathrm{M}$ ABA and incubated for $24 \mathrm{~h}$. Chromatin immunoprecipitation (ChIP) assays using anti-H3ac and anti-H4ac antibodies were performed. The indicated genomic regions (see Fig. 5) were analyzed by ChIP-quantitative PCR. Three independent biological replicates were averaged, and statistical significance of the measurements was analyzed by analysis of variance (ANOVA) (one-way ANOVA with Fisher's post hoc test, ${ }^{\star} P<0.05$ ). Different letters represent a significant difference at $P<0.05$. Bars indicate the standard error of the mean. $\mathbf{c}$ H3ac levels at the ROP loci in 35S:HDA15-GFP and 35S:HDA15-GFP/myb96-1. d Transcript accumulation of ROP genes in wild type, 35S:HDA15GFP, and 35S:HDA15-GFP/myb96-1. In c, d, 2-week-old seedlings grown under LDs were harvested. Statistically significant differences between the wildtype and transgenic plants (Student's $t$ test, ${ }^{\star} P<0.05$ ) are indicated by asterisks. Source data are provided as a Source Data file

study, we found that, while MYB96 alone is sufficient to activate ABA-inducible genes such as $R D$ and $K C S$ genes, MYB96 requires HDA15 to inhibit the expression of ABA-repressible genes. In particular, the MYB96-HDA15 complex selectively represses the $R O P$ genes, which are negative regulators of $\mathrm{ABA}$ signaling.

Given the synergistic interactions between MYB96 and HDA15, the HDA15 protein most likely has regulatory targets non-overlapping with MYB96 in the control of ABA responses, although HDA15 target genes are elusive so far. HDA15 alone can confer ABA sensitivity independently of MYB96. Probably, each individual member deals with mild $\mathrm{ABA}$ responses, but the physical interaction between MYB96 and HDA15 enables to induce strong and robust $\mathrm{ABA}$ responses by regulating upstream core components. 
a

Reporter

pMin35S

Promoter

Mutated promoter
Effector

p35S:MYB96

MYB96

Nos-T

b

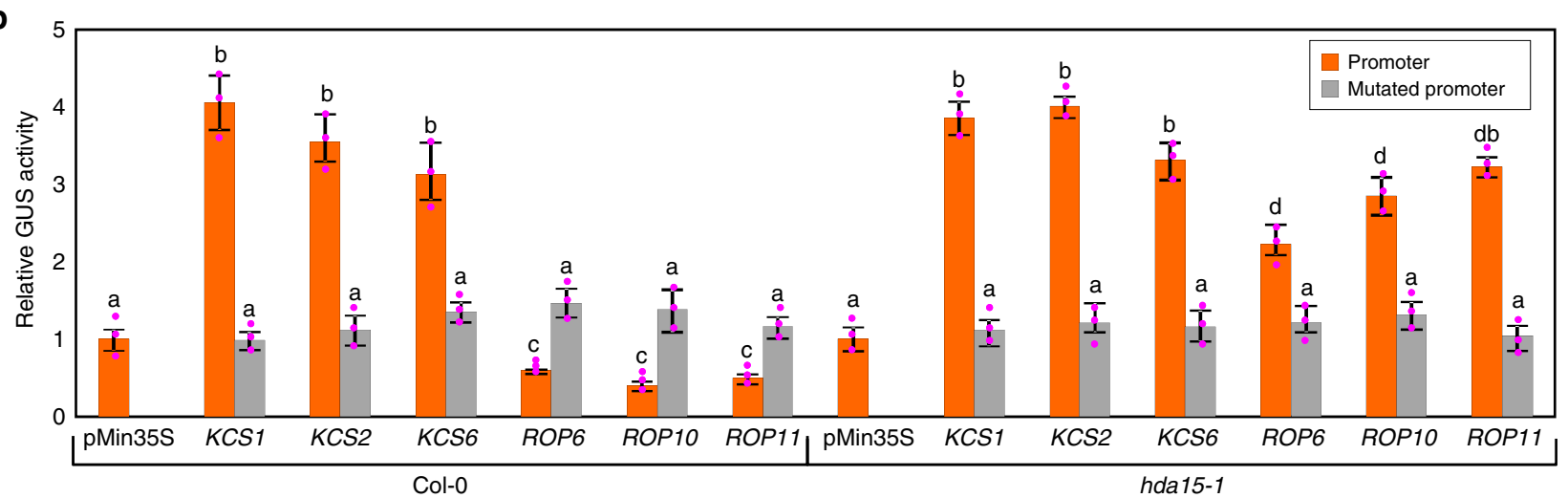

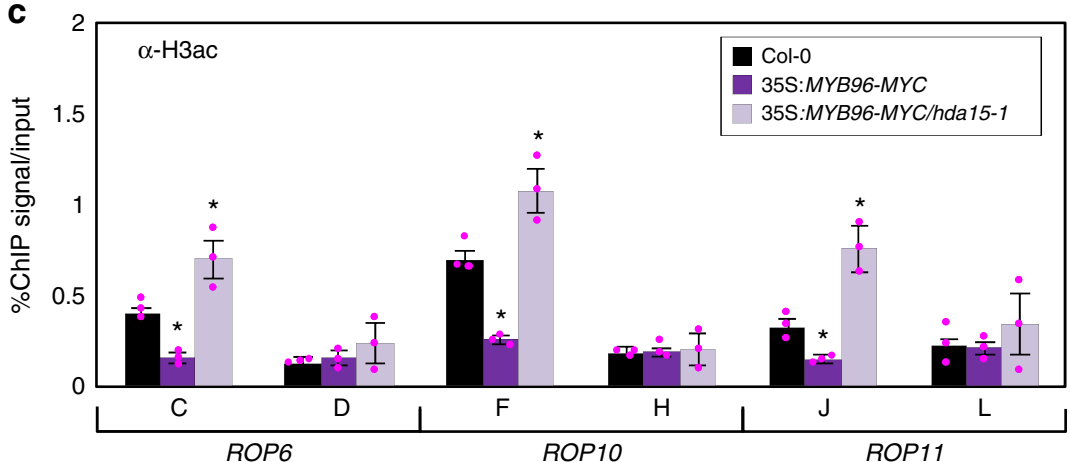

d

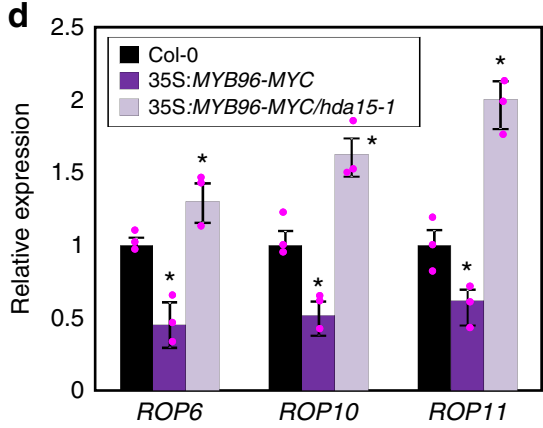

Fig. 7 MYB96 represses expression of ROP genes in an HDA15-dependent manner. a Schematic representation of reporter and effector constructs. The pmKCS and pmROP constructs contain mutated core cis-elements (Supplementary Table 3), which impair recognition of R2R3-type MYB DNA-binding domain, on the KCS and ROP gene promoters, respectively. pMin35S, minimal 35S promoter, CaMV Cauliflower mosaic virus, Nos-T nopaline synthase terminator. $\mathbf{b}$ Transient expression assays using Arabidopsis protoplasts. The recombinant reporter and effector constructs were coexpressed transiently in Arabidopsis protoplasts, and GUS activity was determined fluorimetrically. Luciferase gene expression was used to normalize GUS activity. The normalized values in control protoplasts were set to 1 and represented as relative activation. Three independent measurements were averaged. Different letters represent a significant difference at $P<0.05$ (one-way analysis of variance with Fisher's post hoc test). Bars indicate the standard error of the mean. $\mathbf{c} H 3$ ac levels in 35S:MYB96-MYC/hda15-1. Two-week-old seedlings grown under long days were used to perform chromatin immunoprecipitation (ChIP) using anti-H3ac antibody. The indicated genomic regions (see Fig. 5) were analyzed by ChIP-quantitative PCR. Biological triplicates were averaged, and statistically significant differences between the wild-type and transgenic plants (Student's $t$ test, ${ }^{\star} P<0.05$ ) are indicated by asterisks. $\mathbf{d}$ Expression of $R O P$ genes in 35S:MYB96-MYC/hda15-1. Source data are provided as a Source Data file

One remaining question is how HDA15 selectively represses the ROP genes without affecting MYB96-activated genes. Physical interactions with additional ABA signaling components or transfactors might be involved. Moreover, the strength of MYB96 activation may also be involved. MYB96 activation above the low threshold allows it to mainly act as a transcriptional activator to promote genes contributing to stress tolerance in plants. At a high level of MYB96 activation, a significant portion of MYB96 proteins above a high threshold may get a chance to interact with HDA15, which is also significantly activated under harsh stress conditions, to additionally repress negative regulators of $\mathrm{ABA}$ signaling, further enhancing $\mathrm{ABA}$ responses. In support, MYB96-HDA15 interactions are profoundly enhanced at high concentrations of ABA. Taken together, MYB96 is most likely a master regulator of $\mathrm{ABA}$ signaling. This transcription factor not only activates positive regulators of ABA signaling but also represses repressors of $\mathrm{ABA}$ signaling, ensuring full activation of ABA responses.

HDA15 function is shaped by light conditions. HDA15 interacts with PHYTOCHROME INTERACTING FACTOR 1 (PIF1) and PIF $3^{42,43}$, which act as negative regulators in light responses and repress light-mediated cotyledon development, chlorophyll biosynthesis, and hormonal signaling in the dark ${ }^{65-70}$. The HDA15-PIF complexes co-bind to the target genes and reduce histone $\mathrm{H} 3$ acetylation levels, repressing expression in the $\operatorname{dark}^{42,43}$. Upon light exposure, active forms of phytochromes translocate into the nucleus and induce phosphorylation-induced degradation of PIFs, leading to the dissociation of HDA15 from chromatin and activation of the light-responsive genes ${ }^{42,43}$.

Active roles of HDA15 in light have also been demonstrated. NF-YC1, NF-YC3, NF-YC4, and NF-YC9 interact with HDA15 in the light, and they co-target the promoters of a set of hypocotyl 


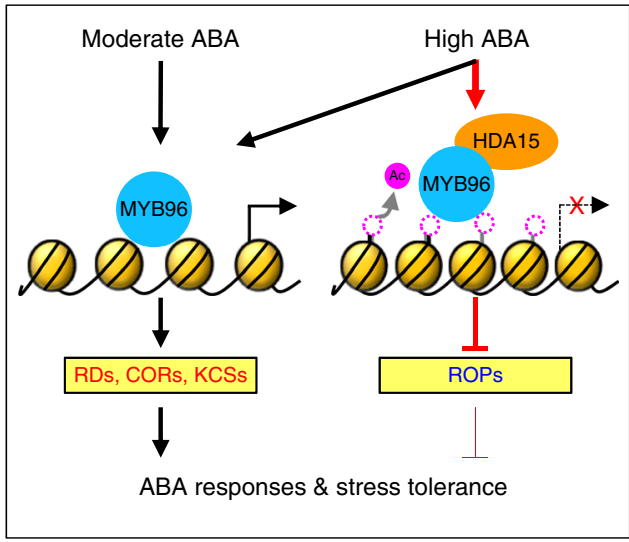

Fig. 8 Proposed working model. In the presence of abscisic acid ( $A B A$ ), MYB96 alone is sufficient to promote expression of ABA-induced genes, including KCS genes. However, MYB96 requires HDA15 to repress the ABA-repressed genes ROP6, ROP10, and ROP11. MYB96 interacts with HDA15 especially at high concentrations of ABA. MYB96 and HDA15 bind to the $R O P$ promoters and repress expression by promoting $\mathrm{H} 3$ and $\mathrm{H} 4$ deacetylation. MYB96 confers strong ABA responses by simultaneously activating positive regulators and suppressing negative regulators of $A B A$ signaling

elongation-related genes, such as INDOLE-3-ACETIC ACID INDUCIBLE 19 (IAA19) and XYLOGLUCAN ENDOTRANSGLUCOSYLASE/HYDROLASE 17 (XTH17), to repress H4 acetylation levels at the associated regions. As a consequence, hypocotyl elongation is suppressed in light ${ }^{71,72}$. However, in the dark, HDA15 dissociates from the target genes, resulting in increased H4ac levels and thereby etiolated growth ${ }^{71}$.

The interactions between HDA15 and MYB96 might also be dependent on light conditions. Consistent with the fact that efficient $\mathrm{ABA}$ responses are required during the daytime, when plants are usually subjected to water deficit, many $A B A$ signaling mediators, including MYB96, are activated in light conditions ${ }^{30,73,74}$. MYB96-HDA15 interaction might be relevant during the day to facilitate activation of drought tolerance by repressing negative regulators of ABA signaling. Plants need to ensure a perfect balance between water loss and photosynthesis $^{75,76}$. The point of crosstalk between light and stress responses not only maximizes metabolic efficiency and growth but also establishes an efficient way to increase stress tolerance under environmentally unfavorable conditions.

\section{Methods}

Plant materials and growth conditions. Arabidopsis thaliana (Columbia-0 ecotype) was used for all experiments unless otherwise specified. Plants were grown under long-day conditions (LDs; 16-h light/8-h dark cycles) with cool white fluorescent light $(120 \mu \mathrm{mol}$ photons $/ \mathrm{m} / \mathrm{s})$ at $22-23^{\circ} \mathrm{C}$. myb96-ox, myb96-1, hda151, 35S:MYB96-MYC, and 35S:HDA15-GFP were previously reported ${ }^{10,12,43}$. To produce transgenic plants overexpressing the MYB96 gene, full-length cDNA was subcloned into the modified binary pBA002 vector under the control of the CaMV 35S promoter ${ }^{77}$. Agrobacterium tumefaciens-mediated Arabidopsis transformation was then performed. myb96-1hda15-1, pMYB96:MYB96-MYC $x$ pHDA15:HDA15-GFP, 35S:MYB96-MYC/hda15-1, and 35S:HDA15-GFP/myb96-1 plants were obtained by genetic crosses.

Seed germination assays. All seeds were harvested at the same time and dried at least 1 month for each genotype. For seed germination assays, seeds for each genotype ( $>40$ seeds per replicate) were sterilized and stratified for 2 days at $4{ }^{\circ} \mathrm{C}$ in darkness. Stratified seeds were transferred to a culture room at $23^{\circ} \mathrm{C}$ under LDs and germinated on MS medium (half-strength MS salts, 0.05\% MES, pH 5.7 and $1 \%$ agar) supplemented with various concentrations of ABA. To measure seed germination percentage, radicle emergence from the seed coat and endosperm was used as a phenotypic marker and counted at regular intervals. Each germination assay was performed in biological triplicates.
ABA treatment and drought. For ABA treatment, 2-week-old seedlings grown under LDs were transferred to half-strength MS-liquid medium supplemented with $20 \mu \mathrm{M}(+)$-cis,trans-ABA (L06278) (Alfa Aesar, Ward Hill, MA, USA). Drought stress was induced in 2-week-old plants grown in soil under LD conditions by halting watering. To prevent direct air-drying of seedlings, small pores were made in the plastic cover 7 days after the start of drought conditions, and the cover was removed 7 days later. Survival rates were measured for each group of plants. Three biologically independent measurements of at least 30 plants were averaged.

RT-qPCR analysis. Total RNA samples were extracted from whole seedlings using TRI reagent (TAKARA Bio, Singa, Japan) according to the manufacturer's recommendations. Total RNA samples were pretreated with an RNAse-free DNAse to eliminate genomic DNA contamination. The first-strand cDNA was synthesized from $2 \mu \mathrm{g}$ of total RNA by reverse transcription using Moloney Murine Leukemia Virus reverse transcriptase (Dr. Protein, Seoul, Korea) with oligo(dT18).

RT-qPCR experiments were performed on the Step-One Plus Real-Time PCR System (Applied Biosystems). The PCR primers used are listed in Supplementary Table 1 . The comparative $C_{\mathrm{T}}$ method was used to determine the relative gene expression with the expression of the EUKARYOTIC TRANSLATION INITIATION FACTOR 4A1 (eIF4A) gene (At3g13920) as an internal control. All RT-qPCR reactions were performed using three independent replicate samples. The specificity of RT-qPCR results was determined by melt curve analysis of the amplified products using the standard method.

Y2H assays. For $\mathrm{Y} 2 \mathrm{H}$ assays, the expression vectors and yeast strains used were supplied by the BD Matchmaker system (Clontech, Mountain View, CA, USA). The yeast strain AH109 harboring the LacZ and His reporter genes was used. PCR products were subcloned into the pGBKT7 (for GAL4 BD fusion) and pGADT7 (for GAL4 AD fusion) vectors. The recombinant constructs were cotransformed into AH109 cells, and the transformed cells were grown on SD/-Leu/-Trp medium and SD/-Leu/-Trp/-His/-Ade medium. The interaction strength was quantified by measuring $\beta$-galactosidase $(\beta$-Gal) activity using $o$-nitrophenyl- $\beta$-D-galactopyranoside (ONPG) as a substrate.

BiFC assays. The full-size MYB96 and HDA15 coding sequence were fused inframe to the 5' end of a gene sequence encoding the C-terminal half of EYFP in the pSATN-cEYFP-C1 vector (E3082) or the N-terminal half of EYFP in the pSATNnEYFP-C1 vector $(E 3081)^{78}$. The IDD14-RFP construct was used as a nucleus localization marker ${ }^{79}$. The recombinant constructs were co-transfected into Arabidopsis mesophyll protoplasts by the PEG-calcium transfection method. Transformed protoplasts were incubated at $23^{\circ} \mathrm{C}$ for $12-16 \mathrm{~h}$ in darkness. Emitted fluorescence was monitored by Zeiss LSM510 confocal microscope (Carl Zeiss, Jena, Germany).

ChIP assays. Harvested plant materials were cross-linked with $1 \%$ formaldehyde, ground in liquid nitrogen, and solubilized in NLB buffer (50 mM HEPES, pH 7.5, $150 \mathrm{mM} \mathrm{NaCl}, 0.5 \mathrm{mM}$ EDTA, $1 \%$ Triton X-100, 0.1\% sodium deoxycholate, $0.1 \%$ sodium dodecyl sulfate (SDS), $1 \mu \mathrm{g} / \mathrm{mL}$ pepstatin A, $1 \mu \mathrm{g} / \mathrm{mL}$ aprotinin, $1 \mu \mathrm{g} / \mathrm{mL}$ leupeptin, and $1 \mathrm{mM}$ phenylmethylsulfonyl fluoride). Chromatin was fragmented on ice with a probe sonicator to obtain approximately 200-800-bp fragments. After sonication, the suspension was centrifuged twice for $20 \mathrm{~min}$ at $13,200 \times g$. The extract was precleared with $100 \mu \mathrm{L}$ of salmon sperm DNA/protein A agarose beads (16-157, Millipore, Billerica, USA) for $1 \mathrm{~h}$. The precleared supernatant was incubated overnight with $10 \mu \mathrm{L}$ of corresponding antibodies, including anti-MYC (05724, Millipore; 1:500 dilution), anti-H3ac (06-599, Millipore; 1:500 dilution), antiH4ac (06-866, Millipore; 1:500 dilution), anti-GFP (ab290, Abcam, Cambridge, USA; 1:500 dilution), or no antibody (control). Then the supernatant was mixed with $100 \mu \mathrm{L}$ of salmon sperm DNA/protein A agarose beads and incubated for $3 \mathrm{~h}$ on a rotating wheel. The beads were washed with $10 \mathrm{~mL}$ of NLB buffer, $1 \mathrm{~mL}$ of low-salt buffer (20 mM Tris-Cl, pH 8, $150 \mathrm{mM} \mathrm{NaCl}, 2 \mathrm{mM}$ EDTA, $1 \%$ Triton X100 , and $0.1 \%$ SDS), $1 \mathrm{~mL}$ of high-salt buffer (20 mM Tris-Cl, pH 8, $500 \mathrm{mM} \mathrm{NaCl}$, $2 \mathrm{mM}$ EDTA, $1 \%$ Triton X-100, and $0.1 \%$ SDS), and $1 \mathrm{~mL}$ of LiCl buffer $(10 \mathrm{mM}$ Tris-Cl, pH 8, 0.25 M LiCl, 1 mM EDTA, 1\% sodium deoxycholate, and $1 \%$ Nonidet P-40). DNA was purified using phenol/chloroform/isoamyl alcohol and sodium acetate ( $\mathrm{pH}$ 5.2). The levels of precipitated DNA fragments were quantified by qPCR using specific primer sets (Supplementary Table 2). Values were normalized according to input DNA levels. Values for control plants were set to 1 after normalization against eIF4a for qPCR analysis.

Transient expression assays. For transient expression assays using Arabidopsis protoplasts, reporter and effector plasmids were constructed. The reporter plasmid (modified pCAMBIA1305 vector) contains a minimal 35S promoter sequence and the GUS-coding gene ${ }^{80}$. The core cis-elements on the KCS and ROP promoters were inserted into the reporter plasmid. pmKCS and pmROP mutant constructs were generated with mutations within core cis-elements (Supplementary Table 3). To construct effector plasmids, MYB96 and HDA15 cDNAs were inserted into the effector vector pBA002 containing the CaMV 35 S promoter.

Fifth leaves were cut into $0.5-\mathrm{mm}$ strips with a fresh razor blade and digested with gentle shaking in an enzyme solution containing $1 \%(\mathrm{w} / \mathrm{v})$ cellulose RS, $0.1 \%$ 
(w/v) macerozyme R10 (Karlan Biochemicals, Cottonwood AZ), 0.6 M mannitol, $10 \mathrm{mM}$ MES (pH 5.7), $1 \mathrm{mM} \mathrm{CaCl}_{2}, 1 \mathrm{mM} \mathrm{MgCl}_{2}, 10 \mathrm{mM} \beta$-mercaptoethanol, and $0.1 \%$ bovine serum albumin $(\mathrm{w} / \mathrm{v})$ for $3 \mathrm{~h}$ at room temperature. The enzyme solution was sieved through a $70-\mu$ m nylon mesh (Carolina Biologicals, Burlington, $\mathrm{NC}$ ) and spun at $45 \times g$ for $5 \mathrm{~min}$. The pellet was washed twice and re-suspended in wash solution (0.6 M mannitol, $4 \mathrm{mM}$ MES, pH 5.7). Recombinant reporter and effector plasmids were cotransformed into Arabidopsis protoplasts by polyethylene glycol-mediated transformation. After 16-h incubation in the dark at $23^{\circ} \mathrm{C}$, transformed protoplasts were processed for quantifying $\beta$-glucuronidase (GUS) activity. A CaMV $35 \mathrm{~S}$ promoter-luciferase construct was also cotransformed as an internal control. The luciferase assay was performed using the Luciferase Assay System Kit (Promega, Madison, WI).

Co-IP assays. A. tumefaciens cells containing 35S:MYB96-MYC and 35S:HDA15GFP constructs were infiltrated to 3 -week-old $N$. benthamiana leaves. Tobacco leaves were homogenized in liquid nitrogen, and total proteins were extracted in protein extraction buffer $(25 \mathrm{mM}$ Tris- $\mathrm{HCl}, \mathrm{pH} 7.5,150 \mathrm{mM} \mathrm{NaCl}, 5 \%$ glycerol, $0.05 \%$ Nonidet P-40, $2.5 \mathrm{mM}$ EDTA, $1 \mathrm{mM}$ phenylmethylsulfonyl fluoride, and $1 \times$ complete cocktail of protease inhibitors). After protein extraction, $5 \%$ of the extracts were used as input control. The protein extracts were mixed with antiMYC antibodies (05-724, Millipore, Billerica, MA, USA; 1:500 dilution) coupled to Protein-A sepharose beads (Sigma-Aldrich, St Louis, MO, USA) and incubated for $46 \mathrm{~h}$ at $4{ }^{\circ} \mathrm{C}$. The precipitated samples were washed at least four times with the protein extraction buffer and then eluted by $1 \times$ SDS-polyacrylamide gel electrophoresis (PAGE) loading buffer to subject to SDS-PAGE with anti-MYC (1:2000 dilution; Millipore) or anti-GFP antibodies (1:500 dilution; sc-9996, Santa Cruz Biotech., Dallas, TX, USA). Similar protocols were used for Co-IP assays for Arabidopsis plants.

Reporting Summary. Further information on experimental design is available in the Nature Research Reporting Summary linked to this article.

\section{Data availability}

Data supporting the findings of this work are available within the paper and its Supplementary Information files. A reporting summary for this article is available as a Supplementary Information file. The source data underlying Fig. 1a, c-e, 2a-g, 3a-d, $4 \mathrm{a}-\mathrm{c}, 5 \mathrm{a}, \mathrm{b}, 6 \mathrm{a}-\mathrm{d}$, and $7 \mathrm{~b}-\mathrm{d}$, as well as Supplementary Figures $1-12$ are provided as a Source Data file. The datasets generated and analyzed during the current study are available from the corresponding author on reasonable request.

Received: 30 January 2018 Accepted: 6 March 2019

Published online: 12 April 2019

\section{References}

1. Vishwakarma, K. et al. Abscisic acid signaling and abiotic stress tolerance in plants: a review on current knowledge and future prospects. Front. Plant Sci. 8, 161 (2017).

2. Cutler, S. R., Rodriguez, P. L., Finkelstein, R. R. \& Abrams, S. R. Abscisic acid: emergence of a core signaling network. Annu Rev. Plant Biol. 61, 651-679 (2010).

3. Nishimura, N. et al. ABA-Hypersensitive Germination1 encodes a protein phosphatase 2C, an essential component of abscisic acid signaling in Arabidopsis seed. Plant J. 50, 935-949 (2007).

4. Gonzalez-Guzman, M. et al. Arabidopsis PYR/PYL/RCAR receptors play a major role in quantitative regulation of stomatal aperture and transcriptional response to abscisic acid. Plant Cell 24, 2483-2496 (2012).

5. Umezawa, $\mathrm{T}$. et al. Type $2 \mathrm{C}$ protein phosphatases directly regulate abscisic acid-activated protein kinases in Arabidopsis. Proc. Natl Acad. Sci. USA 106, 17588-17593 (2009).

6. Fujita, Y. et al. Three SnRK2 protein kinases are the main positive regulators of abscisic acid signaling in response to water stress in Arabidopsis. Plant Cell Physiol. 50, 2123-2132 (2009).

7. Kulik, A., Wawer, I., Krzywinska, E., Bucholc, M. \& Dobrowolska, G. SnRK2 protein kinases-key regulators of plant response to abiotic stresses. OMICS 15, 859-872 (2011).

8. Nuruzzaman, M., Sharoni, A. M. \& Kikuchi, S. Roles of NAC transcription factors in the regulation of biotic and abiotic stress responses in plants. Front Microbiol 4, 248 (2013).

9. Ambawat, S., Sharma, P., Yadav, N. R. \& Yadav, R. C. MYB transcription factor genes as regulators for plant responses: an overview. Physiol. Mol. Biol. Plants 19, 307-321 (2013).

10. Seo, P. J. et al. The MYB96 transcription factor mediates abscisic acid signaling during drought stress response in Arabidopsis. Plant Physiol. 151, 275-289 (2009).
11. Seo, P. J. \& Park, C. M. MYB96-mediated abscisic acid signals induce pathogen resistance response by promoting salicylic acid biosynthesis in Arabidopsis. New Phytol. 186, 471-483 (2010).

12. Seo, P. J. et al. The MYB96 transcription factor regulates cuticular wax biosynthesis under drought conditions in Arabidopsis. Plant Cell 23, 1138-1152 (2011).

13. Lee, H. G. \& Seo, P. J. The Arabidopsis MIEL1 E3 ligase negatively regulates ABA signalling by promoting protein turnover of MYB96. Nat. Commun. 7, 12525 (2016).

14. Lee, H. G., Park, B.-Y., Kim, H. U. \& Seo, P. J. MYB96 stimulates C18 fatty acid elongation in Arabidopsis seeds. Plant Biotechnol. Rep. 9, 161-166 (2015).

15. Lee, H. G., Lee, K. \& Seo, P. J. The Arabidopsis MYB96 transcription factor plays a role in seed dormancy. Plant Mol. Biol. 87, 371-381 (2015).

16. Meshi, T. \& Iwabuchi, M. Plant transcription factors. Plant Cell Physiol. 36, 1405-1420 (1995).

17. Sakamoto, H. et al. Arabidopsis Cys2/His2-type zinc-finger proteins function as transcription repressors under drought, cold, and high-salinity stress conditions. Plant Physiol. 136, 2734-2746 (2004).

18. Kim, S. H. et al. Phosphorylation of the transcriptional repressor MYB15 by mitogen-activated protein kinase 6 is required for freezing tolerance in Arabidopsis. Nucleic Acids Res. 45, 6613-6627 (2017).

19. Luo, M. et al. HD2C interacts with HDA6 and is involved in ABA and salt stress response in Arabidopsis. J. Exp. Bot. 63, 3297-3306 (2012).

20. Yang, Z. Small GTPases: versatile signaling switches in plants. Plant Cell 14 (Suppl), S375-S388 (2002).

21. Urano, D. \& Jones, A. M. Heterotrimeric G protein-coupled signaling in plants. Annu Rev. Plant Biol. 65, 365-384 (2014).

22. Gu, Y., Wang, Z. \& Yang, Z. ROP/RAC GTPase: an old new master regulator for plant signaling. Curr. Opin. Plant Biol. 7, 527-536 (2004).

23. Craddock, C., Lavagi, I. \& Yang, Z. New insights into Rho signaling from plant ROP/Rac GTPases. Trends Cell Biol. 22, 492-501 (2012).

24. Bloch, D. \& Yalovsky, S. Cell polarity signaling. Curr. Opin. Plant Biol. 16, 734-742 (2013)

25. Li, H., Shen, J. J., Zheng, Z. L., Lin, Y. \& Yang, Z. The Rop GTPase switch controls multiple developmental processes in Arabidopsis. Plant Physiol. 126, 670-684 (2001).

26. Lemichez, E. et al. Inactivation of AtRacl by abscisic acid is essential for stomatal closure. Genes Dev. 15, 1808-1816 (2001).

27. Choi, Y., Lee, Y. \& Hwang, J.-U. Arabidopsis ROP9 and ROP10 GTPases differentially regulate auxin and ABA responses. J. Plant Biol. 57, 245-254 (2014).

28. Li, Z., Kang, J., Sui, N. \& Liu, D. ROP11 GTPase is a negative regulator of multiple ABA responses in Arabidopsis. J. Integr. Plant Biol. 54, 169-179 (2012).

29. $\mathrm{Li}, \mathrm{Z}$. et al. ROP11 GTPase negatively regulates ABA signaling by protecting ABI1 phosphatase activity from inhibition by the ABA receptor RCAR1/PYL9 in Arabidopsis. J. Integr. Plant Biol. 54, 180-188 (2012).

30. Lee, H. G., Mas, P. \& Seo, P. J. MYB96 shapes the circadian gating of ABA signaling in Arabidopsis. Sci. Rep. 6, 17754 (2016).

31. Lee, H. G. \& Seo, P. J. The MYB96-HHP module integrates cold and abscisic acid signaling to activate the CBF-COR pathway in Arabidopsis. Plant J. 82, 962-977 (2015)

32. Fujita, Y. et al. AREB1 is a transcription activator of novel ABRE-dependent ABA signaling that enhances drought stress tolerance in Arabidopsis. Plant Cell 17, 3470-3488 (2005).

33. Hoth, S. et al. Genome-wide gene expression profiling in Arabidopsis thaliana reveals new targets of abscisic acid and largely impaired gene regulation in the abi1-1 mutant. J. Cell Sci. 115, 4891-4900 (2002).

34. Gosti, F. et al. ABI1 protein phosphatase $2 \mathrm{C}$ is a negative regulator of abscisic acid signaling. Plant Cell 11, 1897-1910 (1999).

35. Lee, J. H. \& Kim, W. T. Regulation of abiotic stress signal transduction by E3 ubiquitin ligases in Arabidopsis. Mol. Cells 31, 201-208 (2011)

36. Lee, J. H. et al. DWA1 and DWA2, two Arabidopsis DWD protein components of CUL4-based E3 ligases, act together as negative regulators in ABA signal transduction. Plant Cell 22, 1716-1732 (2010).

37. Zhang, X., Garreton, V. \& Chua, N. H. The AIP2 E3 ligase acts as a novel negative regulator of ABA signaling by promoting ABI3 degradation. Genes Dev. 19, 1532-1543 (2005).

38. Schopfer, P., Bajracharya, D. \& Plachy, C. Control of seed germination by abscisic acid: I. Time course of action in Sinapis alba L. Plant Physiol. 64 822-827 (1979)

39. Zhan, A., Schneider, H. \& Lynch, J. P. Reduced lateral root branching density improves drought tolerance in maize. Plant Physiol. 168, 1603-1615 (2015).

40. Zhang, J., Jia, W., Yang, J. \& Ismail, A. M. Role of ABA in integrating plant responses to drought and salt stresses. Field Crops Res. 97, 111-119 (2006).

41. Zheng, Z. L. et al. Plasma membrane-associated ROP10 small GTPase is a specific negative regulator of abscisic acid responses in Arabidopsis. Plant Cell 14, 2787-2797 (2002). 
42. Gu, D. et al. Identification of HDA15-PIF1 as a key repression module directing the transcriptional network of seed germination in the dark. Nucleic Acids Res. 45, 7137-7150 (2017).

43. Liu, X. et al. PHYTOCHROME INTERACTING FACTOR3 associates with the histone deacetylase HDA15 in repression of chlorophyll biosynthesis and photosynthesis in etiolated Arabidopsis seedlings. Plant Cell 25, 1258-1273 (2013).

44. Chinnusamy, V. \& Zhu, J. K. Epigenetic regulation of stress responses in plants. Curr. Opin. Plant Biol. 12, 133-139 (2009).

45. Kim, J. M., To, T. K., Nishioka, T. \& Seki, M. Chromatin regulation functions in plant abiotic stress responses. Plant Cell Environ. 33, 604-611 (2010).

46. Colaneri, A. C. \& Jones, A. M. Genome-wide quantitative identification of DNA differentially methylated sites in Arabidopsis seedlings growing at different water potential. PLoS ONE 8, e59878 (2013).

47. Dhar, M. K., Vishal, P., Sharma, R. \& Kaul, S. Epigenetic dynamics: role of epimarks and underlying machinery in plants exposed to abiotic stress. Int. J. Genomics 2014, 187146 (2014).

48. Han, S. K. \& Wagner, D. Role of chromatin in water stress responses in plants. J. Exp. Bot. 65, 2785-2799 (2014).

49. Kim, J. M., Sasaki, T., Ueda, M., Sako, K. \& Seki, M. Chromatin changes in response to drought, salinity, heat, and cold stresses in plants. Front. Plant Sci. 6, 114 (2015).

50. Sura, W. et al. Dual role of the histone variant H2A.Z in transcriptional regulation of stress-response genes. Plant Cell 29, 791-807 (2017).

51. Han, Z. et al. AtHD2D gene plays a role in plant growth, development, and response to abiotic stresses in Arabidopsis thaliana. Front. Plant Sci. 7, 310 (2016).

52. Luo, M., Cheng, K., Xu, Y., Yang, S. \& Wu, K. Plant responses to abiotic stress regulated by histone deacetylases. Front. Plant Sci. 8, 2147 (2017).

53. Saez, A., Rodrigues, A., Santiago, J., Rubio, S. \& Rodriguez, P. L. HAB1-SWI3B interaction reveals a link between abscisic acid signaling and putative SWI/ SNF chromatin-remodeling complexes in Arabidopsis. Plant Cell 20, 2972-2988 (2008).

54. Zheng, Y. et al. Histone deacetylase HDA9 negatively regulates salt and drought stress responsiveness in Arabidopsis. J. Exp. Bot. 67, 1703-1713 (2016).

55. Han, S. K. et al. The SWI2/SNF2 chromatin remodeling ATPase BRAHMA represses abscisic acid responses in the absence of the stress stimulus in Arabidopsis. Plant Cell 24, 4892-4906 (2012)

56. Peirats-Llobet, M. et al. A direct link between abscisic acid sensing and the chromatin-remodeling ATPase BRAHMA via core aba signaling pathway components. Mol. Plant 9, 136-147 (2016).

57. Liu, Y. et al. Trithorax-group proteins ARABIDOPSIS TRITHORAX4 (ATX4) and ATX5 function in abscisic acid and dehydration stress responses. New Phytol. 217, 1582-1597 (2017).

58. Chen, L. T. \& Wu, K. Role of histone deacetylases HDA6 and HDA19 in ABA and abiotic stress response. Plant Signal. Behav. 5, 1318-1320 (2010).

59. Song, C. P. et al. Role of an Arabidopsis AP2/EREBP-type transcriptional repressor in abscisic acid and drought stress responses. Plant Cell 17, 2384-2396 (2005).

60. Mehdi, S. et al. The WD40 domain protein MSI1 functions in a histone deacetylase complex to fine-tune abscisic acid signaling. Plant Cell 28, 42-54 (2016).

61. Perrella, G. et al. Histone deacetylase complex1 expression level titrates plant growth and abscisic acid sensitivity in Arabidopsis. Plant Cell 25, 3491-3505 (2013).

62. Sridha, S. \& Wu, K. Identification of AtHD2C as a novel regulator of abscisic acid responses in Arabidopsis. Plant J. 46, 124-133 (2006).

63. Chen, L. T., Luo, M., Wang, Y. Y. \& Wu, K. Involvement of Arabidopsis histone deacetylase HDA6 in ABA and salt stress response. J. Exp. Bot. 61, 3345-3353 (2010).

64. Finkelstein, R. R., Gampala, S. S. \& Rock, C. D. Abscisic acid signaling in seeds and seedlings. Plant Cell 14(Suppl), S15-S45 (2002).

65. Kim, J. et al. Functional characterization of phytochrome interacting factor 3 in phytochrome-mediated light signal transduction. Plant Cell 15, 2399-2407 (2003).

66. Sentandreu, M. et al. Functional profiling identifies genes involved in organspecific branches of the PIF3 regulatory network in Arabidopsis. Plant Cell 23, 3974-3991 (2011).

67. Shin, J. et al. Phytochromes promote seedling light responses by inhibiting four negatively-acting phytochrome-interacting factors. Proc. Natl Acad. Sci. USA 106, 7660-7665 (2009).

68. Shin, J., Park, E. \& Choi, G. PIF3 regulates anthocyanin biosynthesis in an HY5-dependent manner with both factors directly binding anthocyanin biosynthetic gene promoters in Arabidopsis. Plant J. 49, 981-994 (2007).

69. Soy, J. et al. Phytochrome-imposed oscillations in PIF3 protein abundance regulate hypocotyl growth under diurnal light/dark conditions in Arabidopsis. Plant J. 71, 390-401 (2012).
70. Stephenson, P. G., Fankhauser, C. \& Terry, M. J. PIF3 is a repressor of chloroplast development. Proc. Natl Acad. Sci. USA 106, 7654-7659 (2009).

71. Tang, Y. et al. Arabidopsis NF-YCs mediate the light-controlled hypocotyl elongation via modulating histone acetylation. Mol. Plant 10, 260-273 (2017).

72. Myers, Z. A. et al. NUCLEAR FACTOR Y, subunit C (NF-YC) transcription factors are positive regulators of photomorphogenesis in Arabidopsis thaliana. PLoS Genet. 12, e1006333 (2016).

73. Seung, D., Risopatron, J. P. M., Jones, B. J. \& Marc, J. Circadian clockdependent gating in ABA signalling networks. Protoplasma 249, 445-457 (2012).

74. Tang, W. et al. FAR-RED ELONGATED HYPOCOTYL3 and FAR-RED IMPAIRED RESPONSE1 transcription factors integrate light and abscisic acid signaling in Arabidopsis. Plant Physiol. 163, 857-866 (2013).

75. Flexas, J., Bota, J., Galmés, J., Medrano, H. \& Ribas-Carbó, M. Keeping a positive carbon balance under adverse conditions: responses of photosynthesis and respiration to water stress. Physiol. Plant 127, 343-352 (2006).

76. Miyashita, K., Tanakamaru, S., Maitani, T. \& Kimura, K. Recovery responses of photosynthesis, transpiration, and stomatal conductance in kidney bean following drought stress. Environ. Exp. Bot. 53, 205-214 (2005).

77. Zhou, Q. et al. FHL is required for full phytochrome A signaling and shares overlapping functions with FHY1. Plant J. 43, 356-370 (2005).

78. Citovsky, V. et al. Subcellular localization of interacting proteins by bimolecular fluorescence complementation in planta. J. Mol. Biol. 362, 1120-1131 (2006).

79. Seo, P. J., Kim, M. J., Ryu, J.-Y., Jeong, E.-Y. \& Park, C.-M. Two splice variants of the IDD14 transcription factor competitively form nonfunctional heterodimers which may regulate starch metabolism. Nat. Commun. 2, 303 (2011).

80. Park, J. et al. Integration of auxin and salt signals by the nac transcription factor NTM2 during seed germination in Arabidopsis. Plant Physiol. 156, 537-549 (2011).

\section{Acknowledgements}

We thank Dr. Keqiang Wu (National Taiwan University, Taiwan) for kindly providing 35S:HDA15-GFP seeds. This work was supported by the Basic Science Research (NRF2019R1A2C2006915) and Basic Research Laboratory (NRF-2017R1A4A1015620) programs provided by the National Research Foundation of Korea and by the NextGeneration BioGreen 21 Program (PJ01319304) provided by the Rural Development Administration.

\section{Author contributions}

P.J.S. conceived and designed the experiments and wrote the paper. H.G.L. conducted experiments and contributed to the study design under the supervision of P.J.S.

\section{Additional information}

Supplementary Information accompanies this paper at https://doi.org/10.1038/s41467019-09417-1.

Competing interests: The authors declare no competing interests.

Reprints and permission information is available online at http://npg.nature.com/ reprintsandpermissions/

Journal Peer Review Information: Nature Communications thanks Keqiang Wu and other anonymous reviewer(s) for their contribution to the peer review of this work. Peer reviewer reports are available.

Publisher's note: Springer Nature remains neutral with regard to jurisdictional claims in published maps and institutional affiliations.

Open Access This article is licensed under a Creative Commons Attribution 4.0 International License, which permits use, sharing, adaptation, distribution and reproduction in any medium or format, as long as you give appropriate credit to the original author(s) and the source, provide a link to the Creative Commons license, and indicate if changes were made. The images or other third party material in this article are included in the article's Creative Commons license, unless indicated otherwise in a credit line to the material. If material is not included in the article's Creative Commons license and your intended use is not permitted by statutory regulation or exceeds the permitted use, you will need to obtain permission directly from the copyright holder. To view a copy of this license, visit http://creativecommons.org/ licenses/by/4.0/.

(C) The Author(s) 2019 